\title{
ZFP207 controls pluripotency by multiple post-transcriptional mechanisms
}

Sandhya Malla ${ }^{1,2,3} \uparrow$, Devi Prasad Bhattarai ${ }^{1,2,3} \uparrow$, Dario Melguizo-Sanchis ${ }^{1,3}$, Ionut Atanasoai ${ }^{4}$, Paula Groza ${ }^{2,3}$, Ángel-Carlos Román ${ }^{5}$, Dandan Zhu ${ }^{6}$, Dung-Fang Lee ${ }^{6,7,8,9}$, Claudia Kutter ${ }^{4}$, Francesca Aguilo ${ }^{1,2,3 *}$

${ }^{1}$ Department of Medical Biosciences, Umeå University, SE-901 85, Umeå, Sweden

${ }^{2}$ Department of Molecular Biology, Umeå University, SE-901 85, Umeå, Sweden

${ }^{3}$ Wallenberg Centre for Molecular Medicine, Umeå University, SE-901 85, Umeå, Sweden

${ }^{4}$ Department of Microbiology, Tumor and Cell Biology, Karolinska Institute, Science for Life

Laboratory, SE-171 77, Stockholm, Sweden

${ }^{5}$ Department of Biochemistry, Molecular Biology and Genetics, University of Extremadura, 06071, Badajoz, Spain

${ }^{6}$ Department of Integrative Biology and Pharmacology, McGovern Medical School, The University of Texas Health Science Center at Houston, Houston, TX 77030, USA

${ }^{7}$ Center for Precision Health, School of Biomedical Informatics, The University of Texas Health Science Center at Houston, Houston, TX 77030, USA

${ }^{8}$ The University of Texas MD Anderson Cancer Center UTHealth Graduate School of Biomedical Sciences, Houston, TX 77030, USA

${ }^{9}$ Center for Stem Cell and Regenerative Medicine, The Brown Foundation Institute of Molecular Medicine for the Prevention of Human Diseases, The University of Texas Health Science Center at Houston, Houston, TX 77030, USA

*Correspondence to: francesca.aguilo@umu.se

$\dagger$ These authors contributed equally to this work 


\begin{abstract}
The pluripotent state is not solely governed by the action of the core transcription factors Oct4, Sox2, and Nanog, but also by a series of co-transcriptional and post-transcriptional events, including alternative splicing (AS) and the interaction of RNA-binding proteins (RBPs) with defined subpopulations of RNAs. Zinc Finger Protein 207 (ZFP207) is an essential transcription factor for mammalian embryonic development. Here, we employ multiple functional analyses to characterize the role of ZFP207 in mouse embryonic stem cells (ESCs). We find that ZFP207 plays a pivotal role in ESC maintenance, and silencing of Zfp207 leads to severe neuroectodermal differentiation defects. In striking contrast to human ESCs, ZFP207 does not transcriptionally regulate stem cell and neuronal-related genes but exerts its effects by control AS networks and acting as an RBP. Our study expands the role of ZFP207 to maintain ESC identity, and underscores ZFP207 functional versatility with key roles in neural fate commitment.
\end{abstract}

\title{
Short title
}

The RBP ZFP207 regulates mouse stemness 
bioRxiv preprint doi: https://doi.org/10.1101/2021.03.02.433507; this version posted March 2, 2021. The copyright holder for this preprint (which was not certified by peer review) is the author/funder. All rights reserved. No reuse allowed without permission.

\section{Introduction}

Mouse embryonic stem cells (ESCs) are derived from the inner cell mass of the preimplantation blastocyst. These cells exhibit unlimited self-renewal capacity and, under appropriated stimulus, retain the potential to differentiate into the three germ layers (Bradley et al., 1984). Mouse ESCs are a useful model to study early mammalian development as their differentiation potential is more robust than that of ESC-like cells derived from other mammals such as humans, which exhibit primed pluripotency and represent a more advanced embryonic stage (Ginis et al., 2004; Nichols and Smith, 2009).

Zinc finger-containing proteins (ZFN or ZFP for human or mice, respectively) are among the largest family of proteins, commonly containing a minimum of one zinc-finger $(\mathrm{ZnF})$ domain which recognize DNA sequences with high affinity. This family of transcription factors play important roles in a variety of cellular processes including development, cellular differentiation, metabolism and oncogenesis (Cassandri et al., 2017). Although ZNF/ZFPs were initially classified as transcription factors, several studies have highlighted additional functions of ZNFs. For instance, it has been shown that ZFP217 could recruit the methyltransferase-like 3 (METTL3) into an inactive complex and hence, restrict N6-methyladenosine $\left(\mathrm{m}^{6} \mathrm{~A}\right)$ deposition at pluripotency transcripts (Aguilo et al., 2015; Lee et al., 2016). In addition, recent studies identified that $\mathrm{ZnF}$ domains can bind RNA, and many ZNF/ZFPs act as putative RNA-binding proteins (RBPs) (Brannan et al., 2016). Indeed, analysis of quantitative global mRNA-protein interaction approaches identified ZNF207 (also termed BuGZ; Bub3 interacting GLEBS and Zinc finger domain-containing protein) as an RBP, among other ZNFs (Baltz et al., 2012; Castello et al., 2012).

ZNF207 is conserved in eukaryotes. It associates with Bub3 and with spindle microtubules to regulate chromosome alignment (Jiang et al., 2014; Jiang et al., 2015; Toledo et al., 2014). Furthermore, both ZNF207 and Bub3 interact with the spliceosome and are required for interphase RNA splicing (Wan et al., 2015), yet its specific molecular role remains elusive.

In human ESCs, ZNF207 functions as a critical transcription factor by transcriptionally regulating the expression of the pluripotency factor OCT4 (Fang et al., 2018), thereby being implicated in the maintenance of self-renewal and pluripotency. Likewise, ZNF207 has been shown to enhance reprogramming efficiency towards pluripotency (Toh et al., 2016). Alternative splicing (AS), in which splice sites in primary transcripts are differentially selected to produce structurally and functionally distinct mRNAs, plays a critical role in cell fate transitions, development, and disease (Gabut et al., 2011). ZNF207 undergoes AS during somatic cell reprogramming and differentiation of human ESCs, an isoform switch that seems to be required for the generation of induced pluripotent stem cells (iPSC), and it might also be necessary to maintain ESC self-renewal and to induce proper differentiation programs (Fang et al., 2018; Toh et al., 2016).

Here we show that ZFP207 plays an important role in the control of mouse ESC identity by a distinct mechanism, which differs from the one observed in human ESCs. Specifically, in mouse ESCs, ZFP207 does not regulate Oct4 transcription but increases OCT4 protein stability by disrupting ubiquitin-dependent proteasomal degradation. In addition, depletion of Zfp207 results in pluripotency defects and blocks neuroectodermal specification without significant changes in the transcriptome of stem cell and neural genes. ZFP207 regulates the expression of the spliceosome, and silencing of Zfp207 leads to aberrant AS patterns. We further describe ZFP207 as a novel RNA-binding protein (RBP), which might directly affect RNA fate. Taken together, this study uncovers the versatile species-specific roles of ZFP207 and the link to coand posttranscriptional pathways that impact cell-fate decisions of mouse ESCs. 
bioRxiv preprint doi: https://doi.org/10.1101/2021.03.02.433507; this version posted March 2, 2021. The copyright holder for this preprint (which was not certified by peer review) is the author/funder. All rights reserved. No reuse allowed without permission.

\section{Results}

\section{Silencing of $Z f p 207$ impairs proliferation in mouse ESCs}

To explore the function of ZFP207 in mouse ESCs, we analyzed the expression of Zfp207 in retinoic acid (RA)-induced differentiation towards the neural lineage (Fig. 1A) and in spontaneous differentiation of ESCs into the three germ layers by embryoid body (EB) formation (Fig. 1B). Expression of the pluripotency factor Oct4 (also known as Pou5fl) was used to monitor proper cell differentiation. Quantitative PCR with reverse transcription (RTqPCR) revealed that $Z f p 207$ was significantly enriched in ESCs compared to differentiated cells (Fig. 1, A and B), and its expression levels gradually decreased along the course of differentiation, correlating with the decrease of ZFP207 and OCT4 protein levels (Fig. 1, C and D).

To better understand the role of ZFP207 in pluripotency and differentiation, we aimed to generate CRISPR/Cas9-mediated knockout (KO) of Zfp207 in ESCs. We used two different strategies: (i) one sgRNA (sgRNAs \#1) targeting exon 3; and (ii) two distinct single guide RNAs (\#1 and \#2) targeting the region containing exon 3 and exon 9 which included the microtubule-binding region domain of ZFP207 (fig. S1A). After picking and expanding individual clones, Western blot analysis and subsequent Sanger sequencing confirmed that all clones analyzed (94) displayed heterozygosity for Zfp207 (fig. S1, B-E) suggesting that homozygous deletion of ZFP207 is lethal as previously suggested (Blomen et al., 2015). Next, we conducted loss-of-function assays by using two distinct short-hairpin RNAs (shRNAs) against Zfp207 (thereafter referred as knockdown 1 and 2 (KD1 and KD2)), to ensure that the observed phenotype is due to Zfp207 depletion and not due to shRNA off-target effects. Silencing of Zfp 207 gene expression in both KD1 and KD2 ESCs was confirmed by RT-qPCR (Fig. 1E) and by Western blot (Fig. 1F). Depletion of Zfp207 led to a reduced proliferation capacity compared to ESCs transduced with scrambled shRNA control (thereafter referred as control), although to a lesser degree in KD1 than in KD2 ESCs (Fig. 1G; 1.3 and 2-fold, respectively). In addition, KD1 and KD2 ESCs showed a significant 1.5-fold increased apoptotic rate compared to control ESCs (Fig. 1H) whilst no significant differences in the cell cycle profile were detected between the three cell lines (Fig. 1I). Overall, our results indicate that ZFP207 is required for the proper proliferation of mouse ESCs.

\section{ZFP207 maintains stem cell identity}

Zfp207-depleted colonies displayed the typical morphology of differentiating ESCs with flat appearance and undefined colony borders (Fig. 2A). Consistently, we detected reduced metabolic activity in ESC after depletion of Zfp 207 determined by alkaline phosphatase activity assay (Fig. 2A). Specifically, down-regulation of $Z f p 207$ resulted in a significant increase in the percentage of partially differentiated colonies whereas the percentage of undifferentiated colonies was significantly decreased compared to control ESCs (Fig. 2B). Such increase in the percentage of partially and finally differentiated colonies in KD1 and KD2 ESCs is a consequence of impaired ESC function as immunofluorescence analysis revealed that silencing of Zfp 207 leads to a decrease of the pluripotency surface marker SSEA1 (Fig. 2C).

Since ZFP207 regulates OCT4 transcription in human, we next analyzed the expression of the pluripotency marker OCT4 and observed that KD1 and KD2 ESCs expressed lower OCT4 protein levels (Fig. 2D) but not Oct4 mRNA levels (Fig. 2E) compared to control ESCs, indicating that ZFP207 could regulate the expression of OCT4 post-transcriptionally. To test this hypothesis, we treated KD1 and KD2 as well as control ESCs with the protein synthesis inhibitor cycloheximide (CHX). Silencing of Zfp207 led to a decrease in the half-life of OCT4 (Fig. $2 \mathrm{~F}$ and G), suggesting that ZFP207 promotes the stability of this pluripotency factor. In addition, treatment with the proteasome inhibitor MG132 restored OCT4 protein levels in 
bioRxiv preprint doi: https://doi.org/10.1101/2021.03.02.433507; this version posted March 2, 2021. The copyright holder for this preprint (which was not certified by peer review) is the author/funder. All rights reserved. No reuse allowed without permission.

mouse ESCs depleted of Zfp207 (Fig. 2, H and I), indicating that ZFP207 interferes with the turnover of this pluripotency factor. Altogether, these results suggest that ZFP207 contributes to the maintenance of the pluripotent state in mouse ESC by regulating post-transcriptional networks.

\section{Silencing of $Z f p 207$ blocks neuroectodermal differentiation}

We next interrogated the role of ZFP207 during lineage specification by assessing the potential of KD1, KD2 and control mouse ESCs to spontaneously differentiated into EBs recapitulating early mouse embryo development. Zfp207 KD1 and KD2 ESCs were able to form EBs (Fig. $3 \mathrm{~A}$ and fig. S2A). Although the cells remained as solid aggregates, we observed a decrease in the size of both Zfp207 KD1 and KD2 EBs compared to control EBs (Fig. 3B), suggesting intrinsic differences during the differentiation process among the different cell lines.

As expected, while Zfp207 expression was gradually down-regulated during the course of differentiation in control cells, Zfp 207 mRNA levels remained low in both KD1 and KD2 cells (fig. S2B). The expression of the pluripotent genes Nanog and Sox2 decreased rapidly in all the cell lines whereas Oct4 decreased progressively, being its expression higher at the latest stage of the time course differentiation in EBs depleted of Zfp 207 compared to control (fig. S2B). EBs originated from the three-shRNA infected ESCs showed normal levels of the mesodermal (Msx 1 and Brachyury or T) and endodermal (Foxa2 and Sox 17) lineage-specific markers (fig. S2B). However, silencing of Zfp207 impaired ectodermal specification as shown by decreased expression of Pax6 and Sox 11 at day four, and Nestin during the course of differentiation in Zfp207 KD EBs compared to controls (Fig. 3C).

To further investigate whether ZFP207 plays a role in neurogenesis, KD1, KD2 and control ESCs were subjected to neural differentiation (fig. S2C). Noticeable, we were not able to characterize $Z f p 207 \mathrm{KD} 1$ and KD2 cells at later stages as they died at day 6. Similar to RA- and EB-mediated differentiation, Zfp207 expression was down-regulated during the course of neurogenesis in control cells, and Zfp 207 mRNA levels remained low in both KD1 and KD2 cells (fig. S2D). The distinct cell lines did not display major morphological differences up to day five of differentiation but KD1 and KD2 underwent a complete block of neuronal differentiation potential at day six (Fig. 3D). In control cell lines, Nestin- and Tuj1immunopositive neuronal projections appeared at day eight (fig. S2E) and were kept in culture until day twelve (Fig. 3D). We also monitored progression of ESC differentiation toward neural fates by RT-qPCR analysis of neuroectodermal markers. Zfp207-depleted cell lines failed to activate the expression of Pax6, Sox 11 and Nestin (Fig. 3E and fig. S2F), consistent with a stall at early ectodermal differentiation. No defects were found in the expression of Tuj1 at the transcriptional level as the up-regulation of this neuronal marker occurred at later stages when the KD1 and KD2 cells had died (Fig. 3E). Altogether these results indicate that ZFP207 expression is required for proper differentiation towards the neuroectoderm lineage.

\section{ZFP207 does not regulate the ectodermal lineage}

In order to gain insight on the role of ZFP207 in ESC pluripotency, we analyzed the global transcriptome response to Zfp207 depletion (Fig. 4A). RNA-sequencing (RNA-seq) analysis identified 382 and 522 genes that were down-regulated in KD1 and KD2 ESCs, respectively (fold change $>1.5 ; \mathrm{p}<0.05$; Fig. $4 \mathrm{~B}$ and table $\mathrm{S} 1$ ). The differences of number of downregulated genes between the two KDs could result in the more severe phenotype observed in cells transduced with shRNA2 compared to shRNA1 (Fig. 1G). The effect on up-regulation was more robust, whereby 1062 and 1082 genes were up-regulated in KD1 and KD2 ESCs, respectively, compared to control ESCs (Fig. 4B). Gene ontology (GO) analysis of biological processes of common down-regulated genes revealed generic functions which included mitotic sister chromatid segregation among other categories (Fig. 4C). Strikingly, poly(A) ${ }^{+}$RNA 
bioRxiv preprint doi: https://doi.org/10.1101/2021.03.02.433507; this version posted March 2, 2021. The copyright holder for this preprint (which was not certified by peer review) is the author/funder. All rights reserved. No reuse allowed without permission.

binding was amongst the most represented molecular function GO categories (fig. S3A). According to the reported function of ZFP207 in kinetochore-microtubule attachment (Dai et al., 2016), top GO categories for molecular function of common down-regulated genes also included microtubule binding (fig. S3A). We validated these results by performing RT-qPCR analysis of down-regulated mitotic sister chromatid segregation genes (e.g., Cdca8 and Cep57l1; fig. S3B). Up-regulated genes were associated with GO biological processes related to RNA splicing and processing, and positive regulation of transcription (Fig. 4D). Similar to the common down-regulated genes, RNA binding, including poly(A) ${ }^{+}$and mRNA binding, were amongst the most represented categories of $\mathrm{GO}$ molecular function (fig. S3C), suggesting a role of ZFP207 in controlling the expression of putative RBPs, primarily involved in RNA splicing and mRNA processing (fig. S3D).

Depletion of Zfp207 did not lead to aberrant transcriptional programs that could explain the developmental defects. Hence, the core pluripotency regulators Oct4, Nanog and Sox2, were not differentially expressed in KD1 and KD2 ESCs compared to control ESCs (Fig. 4E), and there were no differences in the expression of other ESC genes upon silencing of $Z f p 207$ (Fig. $4, F$ and $G$ ). In addition, we did not find major transcriptional differences in genes associated with the neural lineage that could reflect the stall at early ectodermal differentiation in KD1 and KD2 ESCs (Fig. 4, H and I). This is in striking contrast to what it has been reported in human ESCs where depletion of ZNF207 impairs neuroectodermal specification by transcriptionally regulating the expression of genes associated with the ectoderm lineage (Fang et al., 2018). Noteworthy, in human ESCs the isoforms A and C of ZNF207, retaining the exon 9, are highly abundant compared to mouse ESCs, where the isoforms 1 and 2 of Zfp207, retaining the aforementioned exon 9, are highly expressed in differentiated cells (fig. S3, E and F). Hence, in both species there is an antagonistic switch towards using different isoforms during differentiation which could partially explain the differences observed between mouse and human ESCs.

\section{ZFP207 regulates alternative splicing in mouse ESCs}

AS is a fundamental process that increases proteomic diversity in a species- but not organspecific manner (Barbosa-Morais et al., 2012), and it also plays a central role in the regulation of ESC-specific transcriptional programs (Gabut et al., 2011). ZNF207 has been shown to influence pre-mRNA splicing in cancer cells (Wan et al., 2015). Consistent with this observation, our GO enrichment analysis of genes up-regulated upon Zfp207 silencing revealed significant enrichment of GO terms related to RNA splicing (Fig. 4D and Fig. 5A), and some randomly selected spliceosomal genes (Mbnl2, Rbm3 and $S f 3 b 3)$ were further validated by RTqPCR (Fig. 5B).

We therefore sought to investigate whether ZFP207 could control ESC function by a splicingrelated mechanism. To this end, reads from RNA-seq data were mapped to exon-splice junction's sites in order to elucidate genome-wide differential AS events (DSEs) (Alamancos et al., 2015; Trincado et al., 2018b), including alternative 5' and 3' splice-site selection (A5 or $\mathrm{A} 3$ ), alternative first (AF) and last (AL) exon selection, exons that are spliced in a mutually exclusive manner (MX) or that are skipped (SE), and changes in intron retention (RI) (fig. S4A). Comparisons of AS isoform levels were performed in KD1 and KD2 ESCs versus control shRNA ESCs, and differences in AS isoforms were calculated as the change in percent spliced in (dPSI) (Fig. 5C). Alternative first exon selection and exon skipping appeared to be the most predominant splice events (fig. S4B). Our analysis identified 601 and 910 splicing events in $\mathrm{KD} 1$ and KD2 compared to control corresponding to 419 and 609 genes, respectively (false discovery rate $[\mathrm{FDR}]<0.05$; Fig. 5D and table S2). Alterations in DSEs upon knockdown of Zfp207 were not due to changes in transcription as gene expression levels were not correlated to dPSI (fig. S4C). The overlapped AS genes were significantly enriched in functional 
categories associated with RNA splicing, RNA transport and export from the nucleus, and neurogenesis, among others (Fig. 5E and fig. S4D), suggesting that splicing switches occurring upon silencing of $Z f p 207$ are relevant to the severe blocking defect towards the neural lineage observed in KD1 and KD2 ESCs. DSEs were validated by RT-PCR amplifying AS exons that differ in size and band intensity was assessed in order to estimate the exon-inclusion ratios. Specifically, we validated randomly selected ZFP207-mediated DSEs in control, KD1 and KD2 ESCs (Fig. 5F), including the chromatin regulatory cofactor Mtal (AS1 and AS2); the AS factors Tbx3 and Hnrnpal; the transcription factor Ubpl; the histone chaperone Nasp; the neuronal factors Vegfa, Myef2 and Fxrl; and the translation initiation factor eIF4H. Furthermore, we also interrogated the aforementioned DSEs in neural differentiation, and found that the majority of the splicing switches validated in KD1 and KD2 ESCs were also prevalent in differentiated cells (fig. S4E). Collectively, these data indicate that depletion of Zfp207 elicits aberrant AS patterns that resemble the differentiated cell-like pattern, and consequently influences mouse ESC identity.

\section{ZFP207 is a newly identified RBP}

Because ZFP207 influenced AS and it has been identified as a mRNA binder using a quantitative proteomics approach (Baltz et al., 2012; Castello et al., 2012), we assessed the innate binding propensity of ZFP207 in mouse ESCs by employing a modified in vitro RIP protocol (see methods section). We identified 1,400 transcriptome-wide binding events for ZFP207 in our assay (table S3). In total, 942 of these binding sites resided within coding regions of protein-coding genes (Fig. 6A). Since ZFP207 is located in the nucleus, we also found binding to intronic sequences $(178 / 1,400)$. In addition, we detected binding to untranslated regions (UTRs) $(267 / 1,400)$ and exons of non-coding transcripts $(17 / 1,400)$. It is possible that there are more ZFP207-binding events to nascent RNAs, which might have been missed since most intronic sequences are depleted by this method. The 1,400 ZFP207 binding events were distributed over 1,171 genes. Based on a fold-change weighted by the relative expression levels (referred to as binding score), we identified the strongest binding sites within the Ubp1, Marfl, Sae1, Erp29 and Nsd1 transcripts (Fig. 6B). Surprisingly, three of the top five binding sites (Marf1, Sael and Nsd1) were found over exon-exon junctions (fig. S5A). Such binding events are difficult to envision in vivo, unless ZFP207 is part of the exon-exon junction complex and connects exonic ends directly.

Overlapping of the RAPseq and AS data sets revealed 13.7\% of DSEs that were directly bound by ZFP207 (Fig. 6C), including Mtal, Ubpl, Vegfa, and Fxrl with AS switches occurring upon silencing of Zfp207 that were validated (Fig. 5F). However, we cannot exclude that dysregulated expression of genes encoding spliceosomal factors upon depletion of Zfp207 was a major contributor to ESC-differential AS events.

In order to classify the bound genes into functional groups, we performed a pathway enrichment analysis for biological processes and we found that the 1,171 genes felt into 693 significantly enriched pathways over the whole murine transcriptome (FDR $\leq 0.05)$ (Fig. 6D). Interestingly, GO analysis revealed DNA repair, chromosome organization and chromosome segregation among the most represented categories, suggesting that ZFP207 can also regulate the aforementioned pathways not just by interacting with Bub3 (Jiang et al., 2014; Toledo et al., 2014) but also by directly binding to target transcripts and influencing RNA fate. Noteworthy, GO terms that were affected upon Zfp207 silencing such as mRNA processing, splicing, positive regulation of neuron differentiation, and neuron projection development, were highly enriched. This suggests that in addition to regulating a pluripotent AS signature, ZFP207 controls the ESC state through post-transcriptional gene regulatory mechanisms. Indeed, ZFP207-bound transcripts felt into two major ESC gene signatures, namely the Core and Myc modules, which are highly active in the pluripotent state, whereas the Polycomb repressive 
bioRxiv preprint doi: https://doi.org/10.1101/2021.03.02.433507; this version posted March 2, 2021. The copyright holder for this preprint (which was not certified by peer review) is the author/funder. All rights reserved. No reuse allowed without permission.

(PRC) module, operating in differentiation, was under-represented (fig. S5B) (Kim et al., 2010), again in agreement with the observation that ZFP207 regulates pluripotency by transcriptionalindependent programs. The fact that ZFP207-bound transcripts were enriched in the Myc module, which is active in various cancers and predicts cancer outcome, may reveal additional post-transcriptional regulatory functions of ZFP207 not just in pluripotency but also in human diseases. Depiction of the binding score of the ZFP207-bound transcripts showed similar binding activity pattern within the three modules (fig. S5C).

Because RBPs play important roles not just in mRNA splicing but also in other aspects of the posttranscriptional mRNA fate such as nuclear export, localization or nuclear decay (Ye and Blelloch, 2014), we analyzed the distribution of bulk poly (A) ${ }^{+}$RNAs by Fluorescence in situ hybridization (FISH) using an oligo(dT) probe in scramble control, KD1 and KD2 ESCs. mRNA was accumulated in nuclear poly(A) ${ }^{+}$foci upon silencing of Zfp207 (Fig. 6, E and F), suggesting that ZFP207 might play a role in nuclear export. Although cytoplasmic poly(A) ${ }^{+}$ RNA FISH signals were also brighter in Zfp207-depleted cells compared to that in control cells (Fig. 6, E and G), the intensity of cytoplasmic poly $(A)^{+}$RNA pixel and the nuclear to cytoplasmic ratios were less profound in Zfp 207 knockdown compared to control ESCs (Fig. 6, $\mathrm{G}$ and $\mathrm{H}$ ). In addition, cellular fractionation showed a clear accumulation of nuclear RNA upon silencing of Zfp207 whereas no differences were detected in the amount of RNA in the cytoplasmic fractions of the distinct cell lines (Fig. 6I). Although it remains possible that some mRNA will get exported from the nucleus, these results indicate that at least some particular mRNAs are nuclear retained and largely aggregate into poly $(\mathrm{A})^{+}$foci in Zfp207 KD1 and KD2 ESCs.

In summary, we show that ZFP207 plays an important role in maintaining mouse ESC selfrenewal and neuroectodermal specification by controlling co- and post-transcriptional networks. We propose a model whereby ZFP207 regulates the expression of splicing factors to promote bona fide splicing of a specific set of transcripts that alter cellular fate. Additionally, ZFP207 acts as an RBP to facilitate mRNA nuclear export. Hence, the versatility in regulatory capacities of ZFP207 are important for normal expression of pluripotency and neural commitment genes in order to ensure mouse ESC identity (Fig. 6J).

\section{Discussion}

Previous investigations have focused attention on the properties of ZNF207 as a transcription factor and its function in transcriptional control (Fang et al., 2018). An important aspect of our findings is the observation that ZFP207 regulates mouse ESC maintenance through co- and post-transcriptional mechanisms. On the contrary to as human ESCs, mouse ESCs have a naïve pluripotency with unbiased differentiation capacity, and they have proven to be an invaluable model to study development. Hence, although the human orthologue ZNF207 has been described as a critical regulator for self-renewal and pluripotency elsewhere (Fang et al., 2018), we found divergent functional characteristics between both studies. These dissimilarities may reflect two distinct pluripotent states that represent mouse and human ESCs: naïve and primed, respectively (Ginis et al., 2004). In addition, these differences could arise from opposite AS shifting patterns of Zfp207 and ZNF207 that occur during cellular differentiation of mouse and human ESCs, respectively.

ZFP207 is essential for cellular viability in near-haploid cells (Blomen et al., 2015). In line with this, we were unable to fully disrupt the Zfp207 gene in mouse ESCs using CRISPR/Cas9 technology. Hence, we anticipate that ablation of ZFP207 is incompatible with ESC maintenance. Moreover, our results show that depletion of Zfp207 in ESCs resulted in cell proliferation defects by triggering apoptosis, which further shifts the self-renewal phenotype towards differentiation. Similar to human ESCs, we also found that ZFP207 controlled the 
bioRxiv preprint doi: https://doi.org/10.1101/2021.03.02.433507; this version posted March 2, 2021. The copyright holder for this preprint (which was not certified by peer review) is the author/funder. All rights reserved. No reuse allowed without permission.

expression of the pluripotency factor OCT4. However, such regulation did not occur transcriptionally as Oct4 mRNA levels were not affected upon silencing of Zfp207. OCT4 is target for ubiquitination and degradation, and this post-translational modification plays a critical role in OCT4 expression in ESCs (Xu et al., 2009). Our data suggests that ZFP207 would increase OCT4 protein stability by preventing degradation of OCT4 through the proteasome. Consistently, Jiang et al. (Jiang et al., 2014), reported that ZFP207 functions as a chaperone that binds and stabilizes the spindle assembly checkpoint protein Bub3 by protecting Bub3 from degradation by the proteasome. It remains to be elucidated the mechanism by which ZFP207 protects OCT4 from proteasomal degradation as none of the core pluripotency factors were identified in mass spectrometric analysis of mouse ESCs using ZFP207 as bait (Jiang et al., 2014). However, such study identified the E3 ubiquitin ligase UBR5 as a ZFP207interacting partner, suggesting that ZFP207 could exert a secondary role in controlling proteasomal-mediated degradation.

We assessed the function of ZFP207 during in vitro differentiation to the three germ layers, recapitulating the early events in embryogenesis, by performing EB-directed differentiation. Although EB formation and growth were defective after silencing of $Z f p 207$, we could conclude from these experiments that endodermal and mesoderm specification were normal whereas the neuroectodermal lineage was highly compromised. Indeed, upon neural induction, EBs depleted of $Z f p 207$ failed to efficiently up-regulate neural markers, and neuronal differentiation appeared to stall at an early stage. In contrast to human ESCs, RNA-seq analysis revealed that ZFP207 did not drive the transcription of neuronal gene expression programs and could not explain the blockade of neuroectodermal differentiation upon Zfp207 silencing. However, ZFP207 controlled the transcription of multiple splicing factors which potentially could lead to aberrant AS patterns with critical functions in pluripotency. For instance, we validated the upregulated expression of $M b n l 2, R b m 3$, and $S f 3 b 3$. Accordingly, Mbnl2 and $R b m 3$ have been shown to promote differentiated-cell-specific AS patterns (Han et al., 2013; Xia et al., 2018; Yan et al., 2019). To our knowledge, no specific function of the splicing factor Sf3b3 in ESCs has been described. Global profiling of AS landscape revealed alterations of a broad number of genes. Although such splicing defects could be consequence of an indirect effect, through dysregulation of spliceosome gene expression, we cannot exclude the possibility that ZFP207 also regulates AS in a direct manner, through interaction with spliceosome factors (Jiang et al., 2014). Hence, it has been shown that ZFP207 interacts with the splicing components U2AF65 and SF3a3, and transcriptomic analysis revealed splicing defects after depletion of ZFP207 in cancer cells, although such splicing events were not thoroughly characterized (Wan et al., 2015). Our results expand on these prior observations reporting that ZFP207 is a major splicing regulator that plays a critical role in modulating post-transcriptional networks that influence cell fate.

Functional categories of these AS transcripts included RNA metabolism and neuron differentiation. Interestingly, mitotic spindle midzone assembly was also amongst the top identified GO categories, suggesting that ZNF207 might not only promote spindle assembly by undergoing coacervation and hence, concentrating microtubules and tubulin through ZNF207 droplets (Jiang et al., 2015), but also by an RNA-mediated mechanism. Randomly chosen differentially spliced events have been experimentally validated in this study. Most of them (eight out of ten), displayed a switching to a differentiated cell-like AS pattern upon Zfp207 knockdown, indicating that ZFP207 regulates an AS signature that controls pluripotency. In addition, several of the stem cell switches that we identified, such as Nasp or Tbx3, have been shown to be involved in cancer (Alekseev et al., 2011; Krstic et al., 2020).

By using a modified in vitro RIP protocol, we characterized ZFP207 as a novel RBP. ZFP207 has an annotated C2H2-ZNFs through which RNA binding is facilitated (Brannan et al., 2016), 
and two independent interactome capture studies have retrieved ZFP207 in the mRNA-bound proteome (Baltz et al., 2012; Castello et al., 2012). As the extent of intronic binding events that ZFP207 could potentially have inside the nucleus can be orders of magnitude higher than the ones we identified with the in vitro RIP, it would therefore be tempting to speculate that ZFP207 regulates AS by directly binding to specific transcripts. However, it is also plausible that the RNA-binding activity of ZFP207 provide an additional function as we observed an accumulation of poly(A) ${ }^{+}$foci in KD1 and KD2 ESCs. Interestingly, it has been shown that the poly $(\mathrm{A})^{+}$RNA foci that were accumulated upon depletion of Zfp207 are primarily formed by exosome target mRNAs (Fan et al., 2018). Thus, it is possible that ZFP207 functions in the turnover of poly $(\mathrm{A})^{+}$RNA in general, and in the export of pluripotency and neuroectodermal mRNAs in ESCs. It would be interesting to determine in future studies whether the distinct Zfp207 isoforms target a distinct set of RNAs. If that would be the case, the ESC-specific switch in the exon 9 could drive divergent RNA-metabolism-based programs required for ESC selfrenewal and pluripotency.

Collectively, this study further expands the function of ZFP207 and contributes to our understanding of how co- and posttranscriptional programs ensure gene expression to maintain the ESC state. Given that ZFP207 binds both DNA and RNA, the relative contributions between transcriptional and posttranscriptional networks cannot be discriminated. Hence, we cannot exclude the possibility that ZFP207 may also regulate self-renewal transcriptionally i.e., by regulating the expression of splicing factors. Thus, ZFP207 provides an extra layer of control to fine tune the balance between self-renewal and neuronal lineage commitment in mouse ESCs.

\section{Materials and Methods}

\section{Antibodies}

The following commercially available antibodies were used at the indicated concentrations for western blot: Anti-ZFP207 (Santa Cruz Biotechnology, sc-271943, 1:500), anti- $\beta$-ACTIN (Sigma-Aldrich, A5441, 1:2500), Anti-LAMIN A/C (Abcam ab108922), Anti-OCT3/4 (Santa Cruz Biotechnology, sc8628, 1:2500), Goat Anti-Rabbit IgG H\&L (HRP) (Abcam, ab6721, 1:5000), Goat Anti-Mouse IgG H\&L (HRP) (Abcam, 1:1000, ab6789), Rabbit Anti-Goat IgG H\&L (HRP) (Abcam, 1:5000, ab6741), and Rabbit Anti- goat IgG (HRP) (Abcam, ab6771, 1:5000). For IF staining, we used Anti-SSEA1 (ThermoFisher Scientific, MA5-17042, 1:250), Anti-Nestin (Abcam, ab6142, 1:50), Anti-Tuj1 (Abcam, ab18207, 1:200), Anti-OCT3/4 (Santa Cruz Biotechnology, sc8628, 1:2500), Nucleolin (Abcam, ab50279, 1:130), Goat anti-mouse IgG AF488 (ThermoFisher Scientific, A11029, 1:1000), Goat anti-rabbit IgG AF568 (ThermoFisher Scientific, A11011, 1:1000), Anti-Mouse IgG HRP (Abcam, ab6789, 1:1000), and Donkey Anti-Goat IgG AF594 (ThermoFisher Scientific, A11058, 1:250).

\section{Cell culture}

CCE murine ESCs were cultured on $0.1 \%$ gelatin-coated tissue culture plates under feeder free culture conditions at $37^{\circ} \mathrm{C}$ with $5 \% \mathrm{CO}_{2}$ in a humidified incubator. The media composition consists of Dulbecco's modified Eagle's medium (DMEM) high glucose, $15 \%$ fetal bovine serum (FBS; Gibco), 1\% MEM non-essential amino acids (Sigma-Aldrich), $0.1 \mathrm{mM} 2-\beta$ mercaptoethanol, 1\% L-glutamine (Hyclone) and 1\% penicillin/streptomycin (Gibco) and Leukemia inhibitory factor (LIF; R\&D systems).

\section{Lentiviruses production and generation of $Z f p 207 \mathrm{KD}$ mouse ESCs}

Lentiviral particles were generated by transfecting HEK-293T with the following lentiviral plasmids: i) pLKO.1-Puro containing shRNA1 and shRNA2 against Zfp207 (table S4); ii) the packaging vector pCMV-dR8.2; and iii) the helper plasmid pCMV-VSV-G using jet-PEI (Polyplus) as per the manufacturer's instructions. Lentiviral supernatants were collected after 
bioRxiv preprint doi: https://doi.org/10.1101/2021.03.02.433507; this version posted March 2, 2021. The copyright holder for this preprint (which was not certified by peer review) is the author/funder. All rights reserved. No reuse allowed without permission.

48 hours of incubation and concentrated using Amicon Ultra-15 Centrifugal Filter Units (Merck). Early passage mouse ESCs were transduced with the lentiviral particles in mouse ESC media supplemented with polybrene $(8 \mu \mathrm{g} / \mathrm{ml})$ for 24 hours. After 36 hours, infected cells were treated with puromycin $(2 \mu \mathrm{g} / \mathrm{ml})$ for additional 6 days.

\section{Generation of CRISPR/Cas9 knockout ESCs}

sgRNAs were designed using E-CRISP online tool (http://www.e-crisp.org/ECRISP/aboutpage.html) (table S4) (Ran et al., 2013). All sgRNA-Cas9 plasmids were obtained by ligation (T7 DNA ligase, Fermentas) of annealed complementary oligonucleotides of the 20nucleotides target sequences with the pSpCas9(BB)-2A-Puro (PX459) vector (Addgene plasmid \#62988) digested with BbsI (BpilI) (Thermo Scientific). One day before transfection, mouse ESCs were seeded in a 12 wells-plate at a density of 80,000 cells/well. The day after, cells were transfected using Lipofectamine (Invitrogen) with $0.8 \mu \mathrm{g}$ of Cas 9 expression vector containing the corresponding sgRNAs. After 24 hours, transfected cells were diluted and treated with puromycin to obtain isogenic cell clones. Isogenic cell clones were picked and expanded for 10 to 15 days to identify indels (insertion and deletion). KO were screened with PCR, Sanger sequencing and evaluated by western blot analysis.

\section{EB and RA differentiation assays}

EBs were obtained by growing mouse ESCs in low-attachment dishes in the presence of complete medium (DMEM high glucose, 15\% FBS (Gibco), 1\% MEM non-essential amino acids (Sigma-Aldrich), $0.1 \mathrm{mM} 2-\beta$ mercaptoethanol, $1 \%$ L-glutamine (Hyclone) and 1\% penicillin/streptomycin (Gibco) without LIF at a density of $8.8 \times 10^{4} \mathrm{cells} / \mathrm{cm}^{2}$. Medium was replenished every 48 hours. EBs were harvested for extraction of total RNA, whole cell extract and nuclear protein extraction at the indicated time points. RA differentiation was performed plating mouse ESCs onto tissue culture dishes pre-coated with $0.1 \%$ gelatin at a density of 2.1 $\mathrm{x} 10^{3}$ cells $/ \mathrm{cm}^{2}$ in complete media supplemented with $1 \mu \mathrm{M}$ RA (Sigma-Aldrich) for 6 days. Differentiation media containing RA was replenished every 48 hours.

\section{Neural differentiation assay}

Differentiation towards the neuroectodermal lineage was performed as previously described (Aguilo et al., 2017). Briefly, $5 \times 10^{4}$ cells $/ \mathrm{ml}$ were plated in low-attachment dishes in the presence of complete medium without LIF. Following EB formation for 2 days, EBs were cultured in media containing DMEM/F-12, $0.1 \mathrm{mM}$ 2-mercaptoethanol, N2 supplement (100X) (ThermoFisher Scientific), B27 supplement (50X) (ThermoFisher Scientific), and penicillin/streptomycin (ThermoFisher Scientific) supplemented with $1 \mu \mathrm{M}$ RA (SigmaAldrich) for 4 days to allow neuroectodermal differentiation. On day 6, EBs were transferred onto tissue culture dishes pre-coated with $0.1 \%$ gelatin and neuroectodermal differentiation media was replenished every 48 hours. Differentiated cells were harvested for total RNA, nuclear protein extraction and immunostaining analysis at the indicated time points. Cell cultures were maintained at $37^{\circ} \mathrm{C}$ with $5 \% \mathrm{CO}_{2}$ in a humidified incubator.

\section{Cellular proliferation, cell cycle and apoptosis assays}

Cellular proliferation, cell cycle and apoptosis assays were carried out using a Muse Cell Analyzer (Millipore, Sigma-Aldrich) following the manufacturer's recommendations.

\section{Alkaline Phosphatase Staining}

Alkaline Phosphatase Staining was measured using the Alkaline Phosphatase Detection kit (Stemgent) according to standard protocols from the manufacturer. Brightfield images were obtained using a Zeiss microscope and analysed by ZEN lite software. 
bioRxiv preprint doi: https://doi.org/10.1101/2021.03.02.433507; this version posted March 2, 2021. The copyright holder for this preprint (which was not certified by peer review) is the author/funder. All rights reserved. No reuse allowed without permission.

\section{Immunocytochemistry analysis}

Cells were fixed in $4 \%$ formaldehyde (Sigma-Aldrich) and permeabilized with $0.25 \%$ TritonX-100 (Sigma-Aldrich). Following permeabilization, cells were washed twice with PBS and blocked with 10\% goat serum (Invitrogen) and bovine serum albumin (Hyclone) at RT for 1 hour. Cells were then stained with primary antibodies overnight at $4^{\circ} \mathrm{Cat}$ corresponding dilution as previously indicated and secondary staining was performed using corresponding secondary antibodies. 4', 6-diamino-2-phenylindole (DAPI) was used for DNA nuclear stain. Images were acquired using a Nikon microscope (ZEN lite software) and analysed using ImageJ.

\section{CHX treatment and Proteasome inhibition}

Mouse ESCs were seeded at a density of $1 \times 10^{5}$ cells $/ \mathrm{ml}$ in 6 -well plate and after 24 hours, cells were treated with cycloheximide (CHX) at a final concentration of $50 \mu \mathrm{g} / \mathrm{ml}$ for $0,3,6$, and 9 hours, respectively. For proteasome inhibition, cells were incubated with MG-132 at a final concentration of $10 \mu \mathrm{g} / \mathrm{ml}$ for 0,4 and 8 hours, respectively. Cells were harvested for whole cell extracts at indicated time points and subjected to immunoblotting. Blots were quantified using Image Lab software.

\section{RT-qPCR}

Extraction of total RNA was performed using RNeasy Plus Mini Kit (Qiagen). One $\mu \mathrm{g}$ of total RNA was used for complementary DNA synthesis using RevertAid First Strand cDNA Synthesis kit (Invitrogen) as per the manufacturer's instructions. RT-qPCR was performed in triplicate using the PowerUp SYBR Green Mix (ThermoFisher Scientific) and the primers listed in table S4 on a Quantastudio Real-time PCR instrument (Applied Biosystems). -actin levels were used to normalize input amounts. After each round of amplification, melting curve analysis was carried out in order to confirm the PCR product specificity. Ct values obtained for each gene were analyzed using the $\Delta \Delta \mathrm{Ct}$ method from at least three biological replicates.

\section{RNA-Seq and differential gene expression analysis}

RNAseq library preparation was carried out at Novogene facilities (https://en.novogene.com/) and sequenced using Illumina HiSeq 2500 platform (Illumina) as 150 bp pair-ended reads. FASTQ reads were aligned to ENSEMBL mouse transcriptome using BWA-MEM (Li, 2013). Then, the calculation of normalized reads per transcript was retrieved by Express (Roberts and Pachter, 2013). Thereafter, DEG were obtained by using a test under the assumption of a negative binomial distribution where the variance is linked to the mean via a locally-regressed smooth function of the mean (Anders and Huber, 2010) and p-values were adjusted by estimation of the false discovery rate for multiple hypothesis (Benjamini and Hochberg, 1995). We only considered the genes with TPM $>0$ in at least 2 of the samples. FDR value was calculated with the Benjamini-Hochberg correction.

\section{AS and data analysis}

SUPPA2 was used as pipeline for AS analysis (Patro et al., 2017). Briefly, FASTQ reads from RNAseq experiment were aligned and pseudo quantified to mouse genome (mm10) using Salmon (Trincado et al., 2018a). Splicing events in the mouse genome were obtained using a specific SUPPA2 script from a mouse GTF file. Thereafter, percent splicing inclusion (PSI) values for each event were obtained, and the differential PSI values for each condition was calculated along with a p-value for each event.

\section{RT-PCR}

Total RNA was extracted using the RNeasy Mini Kit (Qiagen). Two $\mu$ g of total RNA was reverse transcribed using the RevertAid First Strand cDNA Synthesis kit (Invitrogen). RT- PCR 
bioRxiv preprint doi: https://doi.org/10.1101/2021.03.02.433507; this version posted March 2, 2021. The copyright holder for this preprint (which was not certified by peer review) is the author/funder. All rights reserved. No reuse allowed without permission.

was performed using the DreamTaq Green Master mix (Thermo Fisher Scientific). The specific primers used for AS are listed in table S4. The ratios between splice variants were determined by densitometry using ImageJ software. The percentage of inclusion was calculated by dividing the area of the inclusion band by the combined area of both inclusion and exclusions bands and multiply by 100 .

\section{Gene Ontology (GO) Analysis}

Gene ontology (GO) analysis was performed using the web tool The Database for Annotation, Visualization and Integrated Discovery (DAVID) (http://david.abcc.ncifcrf.gov/).

\section{Heatmaps}

Heat maps were made using Z-score which was calculated as following: $Z=(X-X a v) / X s d$, where $\mathrm{X}$ is the $\log _{2}$-transformed expression level for a given gene in a specific sample, Xav is the mean of $\log _{2}$-transformed expression values for that gene in all samples, and Xsd is the standard deviation of $\log _{2}$ - transformed expression values for that gene across all samples.

\section{In vitro RIP and data analysis}

cDNA of Zfp207 isoform 3 from mouse ESCs was generated (RevertAid ${ }^{\circledR}$ First Strand cDNA Synthesis Kit) and cloned into a HaloTag backbone as a ZFP207-Halo fusion (primers are described in table S4). Zfp207-Halo plasmid was linearized, in vitro transcribed (MegaScript T7 Transcription kit, Thermo Fisher \#AM1333) and in vitro translated (Wheat Germ in vitro translation kit, Promega \#L4380). The in vitro translated Zfp207-Halo fusion product was incubated with total RNA from mouse ESCs. After this binding reaction, the RBP-RNA complex was purified and the bound RNA eluted for subsequent Illumina library preparation (NEXTFLEX small RNA library preparation kit v3, PerkinElmer \#NOVA-5132-06) and paired-end (40x43 cycles) sequenced on an Illumina NextSeq 500. Sequencing reads were trimmed, adapters and ribosomal RNA reads removed and aligned against the reference genome GRCm38.p6 retrieved from GENCODE (https://www.gencodegenes.org/mouse/) using HISAT2 v2.1.0 with default parameters. Only uniquely mapping reads were further considered. PCR duplicate reads were removed. The libraries of the two replicates, HaloTag and input were read count normalized. Binding events (peaks) were called by using MACS2 v2.2.6 (https://github.com/macs3-project/MACS/wiki/Advanced:-Call-peaks-using-MACS2-

subcommands). Genomic regions enriched after subtraction of Halo and Input signals are kept and further filtered at a FDR $\leq 0.01$, a fold change (FC) enrichment $F C \geq 3$ (Halo) and FC $\geq 2$ (Input) and $\mathrm{RPM} \geq 2$ in both replicates. Only consensus peaks were considered further. Genomic features (R Genomic Features package (v1.36.4) and R Genomic Ranges package (v1.36.1)) extracted and pathway enrichment was conducted using R ClusterProfiler (v3.12.0) to compute the significance of the enrichments and org.Mm.eg.db (v3.8.2) to retrieve Entrez IDs required by ClusterProfiler using the entire murine transcriptome as a background model. Each peak has a reported Binding Score computed as the base 2 logarithm of the product between the $\log _{2} R P M$ and the mean enrichment over the two negative controls.

\section{Subcellular RNA fractionation}

2 million cells were harvested and washed twice with PBS. After centrifugation for 10 minutes at 3,000 rpm, the pellets were resuspended in 2 packed volumes of cytoplasmic lysis buffer (10 $\mathrm{mM}$ Tris $\mathrm{HCl} \mathrm{pH} 7.5,0.15 \% \mathrm{NP}-40,150 \mathrm{mM} \mathrm{NaCl}$ supplemented with proteinase and RNase inhibitors) and mixed well by pipetting up and down 15 times. The mix was then incubated on

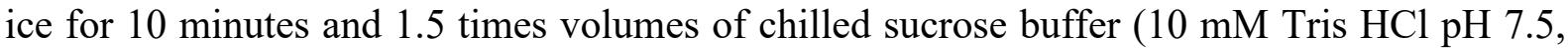
$150 \mathrm{mM} \mathrm{NaCl}, 24 \%$ sucrose) were added to the lysate. The lysate was then centrifuged at $4{ }^{\circ} \mathrm{C}$, $13,000 \mathrm{rpm}$ for 3 minutes, and the supernatant was collected as cytoplasmic fraction. The nuclear pellet was resuspended in 2 packed volumes of cytoplasmic lysis buffer without NP-40 
and mixed with 1.5 times volumes of chilled sucrose buffer followed by centrifugation at 13000 rpm 5 minutes at $4{ }^{\circ} \mathrm{C}$. The washed nuclear fraction was resuspended with chilled glycerol buffer (20 mM Tris HCL pH 7.9, $75 \mathrm{mM} \mathrm{NaCl}, 0.5 \mathrm{mM}$ EDTA and 50\% glycerol) followed by addition of equal volume of cold nuclei lysis buffer (10 mM HEPES pH 7.6, 1 mM DTT, 7.5 $\mathrm{mM} \mathrm{MgCl} 2,0.2 \mathrm{mM}$ EDTA, $0.3 \mathrm{M} \mathrm{NaCl}, 1 \mathrm{M}$ Urea, 1\% NP-40 supplemented with proteinase and RNase inhibitor). The mixture was vortexed twice for 5 seconds each and incubated on ice for 2 minutes followed by centrifugation at high-speed for 5 minutes. The supernatant was collected as a nuclear fraction. Total RNA from cytosolic and nuclear fraction were extracted using the RNeasy Mini Kit (Qiagen).

\section{RNA FISH}

Cells were grown on microscope coverslips placed in tissue culture dishes. After washing twice with PBS, cells were fixed in 4\% paraformaldehyde (Sigma-Aldrich) and permeabilized with $70 \%$ Ethanol for two hours at $4{ }^{\circ} \mathrm{C}$. Following permeabilization, cells were washed twice with $25 \%$ of RNA wash buffer ( $25 \%$ formamide, 2 X SSC) for 5 minutes at RT. Cells were stained with hybridization mix (10\% dextran sulfate, $25 \%$ formamide, $2 \times \mathrm{SSC}, 1 \mathrm{mg} / \mathrm{ml}$ E. coli tRNA, $0.02 \% \mathrm{BSA}, 10 \mathrm{mM}$ vanadyl-ribonucleoside complex) and $2 \mu \mathrm{M} 5^{\prime}$ Cys3-oligo dT(50) probe for 24 hours at $30{ }^{\circ} \mathrm{C}$. The following day, coverslips were washed with $25 \%$ of RNA wash buffer for 60 minutes at $30^{\circ} \mathrm{C}$ and incubated with $20 \mathrm{ng} / \mathrm{ml}$ DAPI (RNAse-free) in $25 \%$ RNA wash 30 minutes at $30^{\circ} \mathrm{C}$. The coverslips were then rinsed with $2 \times \mathrm{SSC}$ buffer followed by RNA equilibration buffer (10 mM Tris $\mathrm{HCl} \mathrm{pH} 7.5,2 \mathrm{x}$ SSC and $0.4 \%$ glucose). Thereafter, coverslips were mounted using anti-bleach buffer $(10 \mathrm{mM}$ trolox, $37 \mathrm{ng} / \mu 1$ glucose oxidase, catalase in RNA equilibration buffer). Images were acquired using a Nikon microscope (ZEN lite software) and analyzed using ImageJ. For quantifying nuclear poly $(\mathrm{A})^{+}$RNA signals $(\mathrm{N})$, images containing at least 30 nuclei were only considered. DAPI staining was masked and overlay on top of the 5'Cys3-oligo d(T) signal, after that intensity of pixel was measured using analyze particles. Then, the total FISH signal was measured as background fluorescence using a threshold setting. And the cytosolic signal was measured as subtracting the nuclear signal from the total signal. All the images were exposed to same magnitude of gain.

\section{Statistical analysis}

Data are shown as mean \pm SEM. GraphPad Prism version 8.0.0 for Windows was used to perform the statistical analysis (GraphPad Software, La Jolla California USA, www.graphpad.com). The significance was determined using student's $t$-test, the ordinary oneway ANOVA and ratio paired $t$-test where indicated including correction for multiple comparison. Probability values of $* \mathrm{p}<0.05, * * \mathrm{p}<0.001, * * * \mathrm{p}<0.0001$ were considered as statistically significant.

\section{References}

Aguilo, F., Zakirova, Z., Nolan, K., Wagner, R., Sharma, R., Hogan, M., Wei, C., Sun, Y., Walsh, M.J., Kelley, K., et al. (2017). THAP1: Role in Mouse Embryonic Stem Cell Survival and Differentiation. Stem Cell Reports 9, 92-107.

Aguilo, F., Zhang, F., Sancho, A., Fidalgo, M., Di Cecilia, S., Vashisht, A., Lee, D.F., Chen, C.H., Rengasamy, M., Andino, B., et al. (2015). Coordination of m(6)A mRNA Methylation and Gene Transcription by ZFP217 Regulates Pluripotency and Reprogramming. Cell Stem Cell 17, 689-704.

Alamancos, G.P., Pages, A., Trincado, J.L., Bellora, N., and Eyras, E. (2015). Leveraging transcript quantification for fast computation of alternative splicing profiles. RNA 21, 15211531. 
bioRxiv preprint doi: https://doi.org/10.1101/2021.03.02.433507; this version posted March 2, 2021. The copyright holder for this preprint (which was not certified by peer review) is the author/funder. All rights reserved. No reuse allowed without permission.

Alekseev, O.M., Richardson, R.T., Tsuruta, J.K., and O'Rand, M.G. (2011). Depletion of the histone chaperone tNASP inhibits proliferation and induces apoptosis in prostate cancer PC-3 cells. Reprod Biol Endocrinol 9, 50.

Anders, S., and Huber, W. (2010). Differential expression analysis for sequence count data. Genome Biology 11, R106.

Baltz, A.G., Munschauer, M., Schwanhausser, B., Vasile, A., Murakawa, Y., Schueler, M., Youngs, N., Penfold-Brown, D., Drew, K., Milek, M., et al. (2012). The mRNA-bound proteome and its global occupancy profile on protein-coding transcripts. Mol Cell 46, 674-690. Barbosa-Morais, N.L., Irimia, M., Pan, Q., Xiong, H.Y., Gueroussov, S., Lee, L.J., Slobodeniuc, V., Kutter, C., Watt, S., Colak, R., et al. (2012). The evolutionary landscape of alternative splicing in vertebrate species. Science 338, 1587-1593.

Benjamini, Y., and Hochberg, Y. (1995). Controlling the False Discovery Rate: A Practical and Powerful Approach to Multiple Testing. Journal of the Royal Statistical Society: Series B (Methodological) 57, 289-300.

Blomen, V.A., Majek, P., Jae, L.T., Bigenzahn, J.W., Nieuwenhuis, J., Staring, J., Sacco, R., van Diemen, F.R., Olk, N., Stukalov, A., et al. (2015). Gene essentiality and synthetic lethality in haploid human cells. Science 350, 1092-1096.

Bradley, A., Evans, M., Kaufman, M.H., and Robertson, E. (1984). Formation of germ-line chimaeras from embryo-derived teratocarcinoma cell lines. Nature 309, 255-256.

Brannan, K.W., Jin, W., Huelga, S.C., Banks, C.A., Gilmore, J.M., Florens, L., Washburn, M.P., Van Nostrand, E.L., Pratt, G.A., Schwinn, M.K., et al. (2016). SONAR Discovers RNABinding Proteins from Analysis of Large-Scale Protein-Protein Interactomes. Mol Cell 64, 282293.

Cassandri, M., Smirnov, A., Novelli, F., Pitolli, C., Agostini, M., Malewicz, M., Melino, G., and Raschella, G. (2017). Zinc-finger proteins in health and disease. Cell Death Discov 3, 17071.

Castello, A., Fischer, B., Eichelbaum, K., Horos, R., Beckmann, B.M., Strein, C., Davey, N.E., Humphreys, D.T., Preiss, T., Steinmetz, L.M., et al. (2012). Insights into RNA biology from an atlas of mammalian mRNA-binding proteins. Cell 149, 1393-1406.

Dai, X.X., Xiong, H., Zhang, M., Sun, S., and Xiong, B. (2016). Zfp207 is a Bub3 binding protein regulating meiotic chromosome alignment in mouse oocytes. Oncotarget 7, 3015530165.

Fan, J., Kuai, B., Wang, K., Wang, L., Wang, Y., Wu, X., Chi, B., Li, G., and Cheng, H. (2018). mRNAs are sorted for export or degradation before passing through nuclear speckles. Nucleic Acids Res 46, 8404-8416.

Fang, F., Xia, N., Angulo, B., Carey, J., Cady, Z., Durruthy-Durruthy, J., Bennett, T., Sebastiano, V., and Reijo Pera, R.A. (2018). A distinct isoform of ZNF207 controls selfrenewal and pluripotency of human embryonic stem cells. Nat Commun 9, 4384.

Gabut, M., Samavarchi-Tehrani, P., Wang, X., Slobodeniuc, V., O'Hanlon, D., Sung, H.K., Alvarez, M., Talukder, S., Pan, Q., Mazzoni, E.O., et al. (2011). An alternative splicing switch regulates embryonic stem cell pluripotency and reprogramming. Cell 147, 132-146.

Ginis, I., Luo, Y., Miura, T., Thies, S., Brandenberger, R., Gerecht-Nir, S., Amit, M., Hoke, A., Carpenter, M.K., Itskovitz-Eldor, J., et al. (2004). Differences between human and mouse embryonic stem cells. Dev Biol 269, 360-380.

Han, H., Irimia, M., Ross, P.J., Sung, H.K., Alipanahi, B., David, L., Golipour, A., Gabut, M., Michael, I.P., Nachman, E.N., et al. (2013). MBNL proteins repress ES-cell-specific alternative splicing and reprogramming. Nature 498, 241-245.

Jiang, H., He, X., Wang, S., Jia, J., Wan, Y., Wang, Y., Zeng, R., Yates, J., 3rd, Zhu, X., and Zheng, Y. (2014). A microtubule-associated zinc finger protein, BuGZ, regulates mitotic 
bioRxiv preprint doi: https://doi.org/10.1101/2021.03.02.433507; this version posted March 2, 2021. The copyright holder for this preprint (which was not certified by peer review) is the author/funder. All rights reserved. No reuse allowed without permission.

chromosome alignment by ensuring Bub3 stability and kinetochore targeting. Dev Cell 28, 268281.

Jiang, H., Wang, S., Huang, Y., He, X., Cui, H., Zhu, X., and Zheng, Y. (2015). Phase transition of spindle-associated protein regulate spindle apparatus assembly. Cell 163, 108-122.

Kim, J., Woo, A.J., Chu, J., Snow, J.W., Fujiwara, Y., Kim, C.G., Cantor, A.B., and Orkin, S.H. (2010). A Myc network accounts for similarities between embryonic stem and cancer cell transcription programs. Cell 143, 313-324.

Krstic, M., Hassan, H.M., Kolendowski, B., Hague, M.N., Anborgh, P.H., Postenka, C.O., Torchia, J., Chambers, A.F., and Tuck, A.B. (2020). Isoform-specific promotion of breast cancer tumorigenicity by TBX3 involves induction of angiogenesis. Lab Invest 100, 400-413.

Lee, D.F., Walsh, M.J., and Aguilo, F. (2016). ZNF217/ZFP217 Meets Chromatin and RNA. Trends Biochem Sci 41, 986-988.

Li, H.W. (2013). Aligning sequence reads, clone sequences and assembly contigs with BWAMEM. arXiv: Genomics.

Nichols, J., and Smith, A. (2009). Naive and primed pluripotent states. Cell Stem Cell 4, 487492.

Patro, R., Duggal, G., Love, M.I., Irizarry, R.A., and Kingsford, C. (2017). Salmon provides fast and bias-aware quantification of transcript expression. Nature Methods 14, 417-419.

Ran, F.A., Hsu, P.D., Wright, J., Agarwala, V., Scott, D.A., and Zhang, F. (2013). Genome engineering using the CRISPR-Cas9 system. Nat Protoc 8, 2281-2308.

Roberts, A., and Pachter, L. (2013). Streaming fragment assignment for real-time analysis of sequencing experiments. Nature Methods 10,71-73.

Toh, C.X., Chan, J.W., Chong, Z.S., Wang, H.F., Guo, H.C., Satapathy, S., Ma, D., Goh, G.Y., Khattar, E., Yang, L., et al. (2016). RNAi Reveals Phase-Specific Global Regulators of Human Somatic Cell Reprogramming. Cell Rep 15, 2597-2607.

Toledo, C.M., Herman, J.A., Olsen, J.B., Ding, Y., Corrin, P., Girard, E.J., Olson, J.M., Emili, A., DeLuca, J.G., and Paddison, P.J. (2014). BuGZ is required for Bub3 stability, Bub1 kinetochore function, and chromosome alignment. Dev Cell 28, 282-294.

Trincado, J.L., Entizne, J.C., Hysenaj, G., Singh, B., Skalic, M., Elliott, D.J., and Eyras, E. (2018a). SUPPA2: fast, accurate, and uncertainty-aware differential splicing analysis across multiple conditions. Genome Biology 19, 40.

Trincado, J.L., Entizne, J.C., Hysenaj, G., Singh, B., Skalic, M., Elliott, D.J., and Eyras, E. (2018b). SUPPA2: fast, accurate, and uncertainty-aware differential splicing analysis across multiple conditions. Genome Biol 19, 40.

Wan, Y., Zheng, X., Chen, H., Guo, Y., Jiang, H., He, X., Zhu, X., and Zheng, Y. (2015). Splicing function of mitotic regulators links R-loop-mediated DNA damage to tumor cell killing. J Cell Biol 209, 235-246.

Xia, W., Su, L., and Jiao, J. (2018). Cold-induced protein RBM3 orchestrates neurogenesis via modulating Yap mRNA stability in cold stress. J Cell Biol 217, 3464-3479.

Xu, H., Wang, W., Li, C., Yu, H., Yang, A., Wang, B., and Jin, Y. (2009). WWP2 promotes degradation of transcription factor OCT4 in human embryonic stem cells. Cell Res 19, 561573.

Yan, J., Goerne, T., Zelmer, A., Guzman, R., Kapfhammer, J.P., Wellmann, S., and Zhu, X. (2019). The RNA-Binding Protein RBM3 Promotes Neural Stem Cell (NSC) Proliferation Under Hypoxia. Front Cell Dev Biol 7, 288.

Ye, J., and Blelloch, R. (2014). Regulation of pluripotency by RNA binding proteins. Cell Stem Cell 15, 271-280. 
bioRxiv preprint doi: https://doi.org/10.1101/2021.03.02.433507; this version posted March 2, 2021. The copyright holder for this preprint (which was not certified by peer review) is the author/funder. All rights reserved. No reuse allowed without permission.

\section{Acknowledgements}

General: We would like to thank Aguilo Lab members for useful discussion, the Biochemical Imaging Centre Umeå for assistance with the imaging processing, and David Muñoz Forcada for help with the figure's presentation.

Funding: This research was supported by grants from the Knut and Alice Wallenberg Foundation, Umeå University, Västerbotten County Council, Swedish Research Council (201701636), and Kempe Foundation (SMK-1766 and JCK-1723.1). D. Z. is supported by DoD Horizon Award (W81XWH-20-1-0389). D.-F.L. is a CPRIT Scholar in Cancer Research and is supported by CPRIT Award RR160019, the Rolanette and Berdon Lawrence Research Award, the Pablove Foundation Childhood Cancer Research Seed Grant Award, and CPRIT UTHealth Cancer Genomic Core pilot grant (CGC-FY20-1).

Author contributions: F.A. conceived and designed the study. S.M., D.P.B. D.M.S and P.G. performed experiments, I.A. and C.K. performed RAP-seq analysis, A.C.R., D.Z. and D.F.L performed bioinformatics analysis. F.A. wrote the manuscript. All authors reviewed and edited the manuscript.

Competing interests: The authors declare that they have no competing interests

Data and materials availability: All next generation sequencing data will can be publicly accessed in ArrayExpress webserver (E-MTAB-10108 and E-MTAB-10113). 
bioRxiv preprint doi: https://doi.org/10.1101/2021.03.02.433507; this version posted March 2, 2021. The copyright holder for this preprint (which was not certified by peer review) is the author/funder. All rights reserved. No reuse allowed without permission.

\section{Figures and Tables}

\section{Figure 1. Depletion of $Z f p 207$ leads to growth defects of mouse ESCs}

(A) RT-qPCR analysis of Zfp207 and Oct4 in mouse ESCs during retinoic acid (RA)-induced and (B) embryoid body (EB)-mediated differentiation. mRNA levels are normalized to $\beta$-Actin and represented as mean \pm SEM relative to the expression at day $0 . \mathrm{n}=3,{ }^{*} \mathrm{p}<0.05,{ }^{* *} \mathrm{p}<$ $0.001, * * * \mathrm{p}<0.0001$. An unpaired Student's $t$-test was used for statistical analysis. (C) Representative western blot of ZFP207 and OCT4 of mouse ESCs during RA-induced and (D) EB-mediated differentiation. $\beta$-ACTIN was used as a loading control. (E) RT-qPCR and western blot (F) to monitor the knockdown efficiency of Zfp207 (sh1 and sh2). Zfp207 is normalized to $\beta$-Actin and represented as mean \pm SEM relative to the expression of the scramble control ( $\mathrm{shScr}$ ). $\mathrm{n}=3, * * * \mathrm{p}<0.0001$. Statistical significance was determined using the ordinary one-way ANOVA. For the immunoblot, $\beta$-ACTIN was used as a loading control. (G) Cell proliferation rate of shScr, sh1 and sh2 ESCs assessed over a period of 6 days. Measurements were taken every other day. The cell proliferation rates are represented as mean $\pm S E M, n=3$, ${ }^{*} \mathrm{p}<0.05, * * * \mathrm{p}<0.0001$. Statistical analysis was performed with an unpaired Student's $t$-test. (H) Percentage of live (Annexin V-) and apoptotic cells (Annexin V+) in sh1 and sh2 mouse ESCs compared to shScr. Data are represented as mean \pm SEM, $n=3, * p<0.05,{ }^{*} * \mathrm{p}<0.01$. An unpaired Student's $t$-test was used for statistical analysis. (I) Cell cycle distribution in sh1 and sh 2 mouse ESCs relative to shScr was examined according to the DNA content index. Data are represented as mean $\pm \mathrm{SEM}, \mathrm{n}=3$

\section{Figure 2. ZFP207 is required for mouse ESCs self-renewal}

(A) Representative images of AP staining of control (shScr) and Zfp207-depleted (sh1 and sh2) mouse ESCs. Scale bars, $20 \mu \mathrm{M}$. (B) Percentage of fully differentiated (FD), partially differentiated (PD) and undifferentiated (UN) ESC colonies in shScr, sh1 and sh2. Data are represented as mean \pm SEM, $\mathrm{n}=3, * \mathrm{p}<0.05, * * \mathrm{p}<0.001$. An unpaired Student's $t$-test was used for statistical analysis. (C) Representative immunofluorescence analysis of SSEA1 in shScr, sh1 and sh2 ESCs. DAPI was used as the nuclear marker. Scale bars, $20 \mu \mathrm{m}$. (D) Representative western blot analysis of OCT4 and ZFP207 in shScr, sh1 and sh2 ESCs. $\beta$ ACTIN was used as a loading control. (E) RT-qPCR analysis of Oct4 in shScr, sh1 and sh2 ESCs. Oct4 is normalized to $\beta$-Actin and represented as mean $\pm \mathrm{SEM}$ relative to $\mathrm{shScr} ; \mathrm{n}=3$. (F) Western blot analysis of OCT4 during a 9-h cycloheximide (CHX) time course treatment in shScr, sh 1 and sh2 ESCs. $\beta$-ACTIN was used as a loading control. (G) Protein degradation curves were made after quantification and normalization of the bands from (F). Data are represented as mean \pm SEM, $\mathrm{n}=3$, $^{*} \mathrm{p}<0.05, * * \mathrm{p}<0.01$. Statistical significance was determined using the ordinary one-way ANOVA. (H) Western blot analysis of OCT4 during 4 and $8 \mathrm{~h}$ treatment with the proteasome inhibitor MG132. $\beta$-ACTIN was used as a loading control and bands were quantified and normalized (I). Data are represented as mean \pm SEM, $n$ $=3, * \mathrm{p}<0.05, * * \mathrm{p}<0.01, \mathrm{~ns}=$ not significative. Statistical significance was determined using the ordinary one-way ANOVA.

\section{Figure 3. Loss of $Z f p 207$ results in defective differentiation}

Representative bright-field images (10x magnification) of embryoid bodies (EB) generated from control (shScr), and Zfp207-depleted ESCs (sh1 and sh2) at day 8 of differentiation. Scale bars, $200 \mu \mathrm{M}$. (B) Quantification of EB size in sh1 and sh2 ESCs compared to shScr. Data are represented as mean $\pm \mathrm{SEM}, \mathrm{n}=3, * * \mathrm{p}<0.001$. An unpaired Student's $t$-test was used for statistical analysis. (C) RT-qPCR analysis of the neural-associated genes (Pax6, Sox11 and Nestin) in shScr, sh1 and sh2 ESCs along the time-course of EB-mediated differentiation. 
mRNA levels are normalized to $\beta$-Actin and represented as mean $\pm \mathrm{SEM}$ relative to the expression of shScr at day $0 ; \mathrm{n}=3, * * \mathrm{p}<0.001$. A ratio paired Student's $t$-test was used for statistical analysis. (D) Immunostaining of NESTIN (green) and TUJ1 (red) in shScr, sh1 and sh2 derived neural progenitors on the indicated days of neuroectodermal differentiation. Nuclei were counterstained with DAPI. Scale bar, $20 \mu \mathrm{M}$. (E) Gene expression of the neural-associated markers (Pax6, Sox11, Nestin, and Tuj1) in shScr, sh1 and sh2 ESCs along the course of directed neural differentiation. Data points for sh1 and sh2 were profiled up to day 5 because the cells died. mRNA levels are normalized to $\beta$-Actin and represented as mean $\pm \mathrm{SEM}$ relative to the expression of shScr at day $0 ; \mathrm{n}=3, * \mathrm{p}<0.05, * * \mathrm{p}<0.001$. An unpaired Student's $t$-test was used for statistical analysis.

\section{Figure 4. Neural transcriptome is not altered upon depletion of $Z f P 207$}

(A) Volcano plots of common differentially expressed genes in control (shScr) and Zfp207depleted (sh1 and sh2) mouse ESCs. The statistically significant $(\mathrm{p}<0.05$; $>1.5$-fold) upregulated (Up) and down-regulated (Down) genes are indicated in red and blue, respectively. Zfp 207 is depicted in black. Grey dots indicate non-significant (NS) and $<1.5$-fold differential expressed genes. (B) Venn diagram depicting the overlap of down-regulated and up-regulated genes between sh1 and sh2 ESCs $(\mathrm{FC}>1.5$ and $\mathrm{p}<0.05)$. (C) Gene ontology (GO) analysis of biological processes associated with common down-regulated and (D) up-regulated genes in Zfp207-depleted ESCs (sh1 and sh2); NHEJ: Non-homologous end joining. (E) RT-qPCR analysis of pluripotency markers (Oct4, Nanog and Sox2) in shScr, sh1 and sh2 ESCs. mRNA expression is normalized to $\beta$-Actin and represented as mean \pm SEM relative to shScr; $\mathrm{n}=3$. (F) Heatmap of column z-scores of $\log 2$ transformed values of genes related to pluripotency in shScr, sh1 and sh2 ESCs. (G) GSEA plots depicting the expression of ESC genes in Zfp207depleted ESCs compared to shScr. High and low expression of genes is represented in red and blue color, respectively. FDR, false discovery rate; NES, normalized enrichment score. (H) Heatmap and (I) GSEA plot showing the global expression of genes related to ectoderm and neural lineage in Zfp207-depleted ESCs compared to shScr. High and low expression of genes is represented in red and blue color respectively. FDR, false discovery rate; NES, normalized enrichment score.

\section{Figure 5. Silencing of $Z f p 207$ leads to AS defects}

(A) Heatmap of column z-scores of $\log 2$ transformed values of genes involved in alternative splicing in control (shScr) and Zfp207-depleted (sh1 and sh2) mouse ESCs. (B) RT-qPCR analysis of Rbm3, Mbnl2 and $S f 3 b 3$ in shScr, sh1 and sh2 ESCs. mRNA expression is normalized to $\beta$-Actin and represented as mean \pm SEM relative to $\operatorname{shScr} ;{ }^{*} \mathrm{p}<0.05,{ }^{* *} \mathrm{p}<0.01$, $* * * \mathrm{p}<0.001, * * * * \mathrm{p}<0.0001, \mathrm{n}=3$. An unpaired Student's $t$-test was used for statistical analysis. (C) Violin plot depicting AS events in each category in mouse ESCs depleted of Zfp207 (sh1 and sh2) related to shScr. A3 (Alternative 3'); A5 (Alternative 5'); AF (Alternative first exon); AL (Alternative last exon); MX (Mutually exclusive exons); RI (Intron retention); SE (Exon skipping). (D) Venn diagram showing the overlap of genes undergoing AS in mouse ESCs infected with sh1 and sh2 against Zfp207. (E) Gene ontology analysis of common genes undergoing AS in mouse ESCs infected with sh1 and sh2 against Zfp207 according to the type of AS event. (F) Representative RT-PCR validations of Zfp207-regulated AS events (Mtal; Tbx3; Ubp1; Vegfa, and Fxr1 (left panel) and Mta1, Hnrnpal, Nasp, Myef2, Eif4H (right panel)). For Mta1, AS1 (alternative splicing 1) and AS2 (alternative splicing 2). \#1 and \#2 indicate distinct biological replicates. *Denotes an isoform which was not taken in consideration for the quantification. The structure of each isoform is indicated (not to scale). Alternative exons are blue. The percent spliced in (PSI) was quantified for each condition. 
bioRxiv preprint doi: https://doi.org/10.1101/2021.03.02.433507; this version posted March 2, 2021. The copyright holder for this preprint (which was not certified by peer review) is the author/funder. All rights reserved. No reuse allowed without permission.

\section{Figure 6. ZFP207 is a novel RBP}

(A) Schematic representation of the RNA Affinity Purification followed by Sequencing (RAPseq) experimental procedure. (B) Barchart representing the distribution of ZFP207 binding sites across common transcript features. (C) Scatterplot depicting ranked distribution of ZFP207 binding sites. The top 5 strongest binding sites are highlighted. (D) Venn diagram showing the overlap of genes retrieved by RAP-seq and genes undergoing AS in mouse ESCs upon Zfp207 knockdown. (E) Bar- and dot plots show significantly enriched GO terms for Biological processes for ZFP207 bound genes. Each term is ranked by the sum of all binding scores of all peaks present in this pathway. Top $30 \mathrm{GO}$ terms are shown. Circle diameter represents the number of genes bound by ZFP207 in that specific pathway. (F) FISH analysis of poly (A) ${ }^{+}$RNA distribution in scramble control (shScr) and Zfp207-depleted ESCs (sh1 and sh2) stained with 5'Cys3-oligo dT(50). Nuclei were counterstained with DAPI. All images were subjected to the same exposure settings. Scale bar, $20 \mu \mathrm{M}$. (G, H) Quantification of nuclear and cytoplasmic poly(A) ${ }^{+}$RNA FISH signals in shScr, sh1 and sh2 respectively. Data are represented as mean $\pm \mathrm{SEM} ; * * * \mathrm{p}<0.001, * * * * \mathrm{p}<0.0001 ; \mathrm{n}=2$. An unpaired Student's $t$ test was used for statistical analysis. (I) Ratio of nuclear/cytoplasmic poly(A) ${ }^{+}$FISH signals in shScr, sh 1 and sh2 mouse ESCs. Data are represented as mean $\pm \mathrm{SEM} ; * \mathrm{p}<0.05, * * * \mathrm{p}<$ $0.001 ; \mathrm{n}=2$. An unpaired Student's $t$-test was used for statistical analysis. (J) Relative nuclear and cytosolic total RNA levels in Zfp207-depleted ESCs compared to shScr. Results are represented as mean $\pm \mathrm{SEM} ; * \mathrm{p}<0.05 ; \mathrm{n}=2$. An unpaired Student's $t$-test was used for statistical analysis. (K) ZFP207 modulates mouse ESCs pluripotency and neuroectodermal differentiation. In our model, ZFP207 regulates the expression of multiple splicing factors and AS pattern, influencing cellular fate specification. Besides, ZFP207 regulates OCT4 stability post-transcriptional networks. In addition, ZFP207 acts as an RBP to promote mRNA nuclear export. Hence, the co- and post-transcriptional regulatory mechanisms controlled by ZFP207 are essential for maintaining proper mouse ESC identity. 
A bioRxiv preprint doi: $\quad$ ths://doi.org/10.1101/2021.03.02.4 3507 ; this version posted March 2, 202 $\mathbf{D}$ The copyright holder for this prep

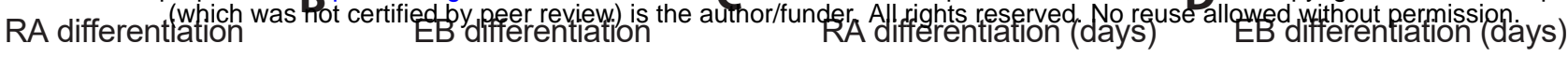
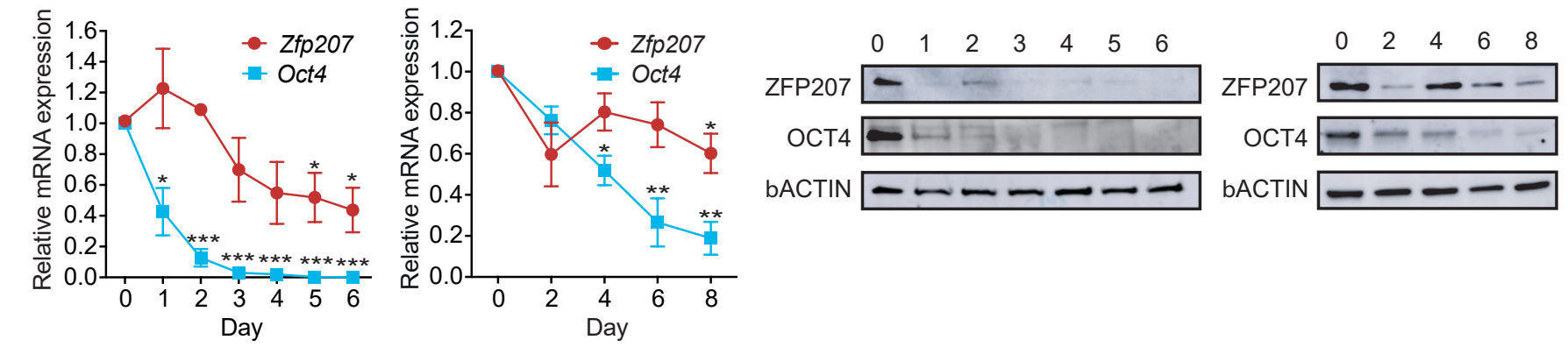

E

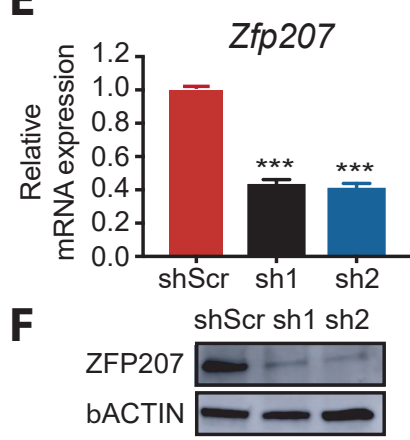

G

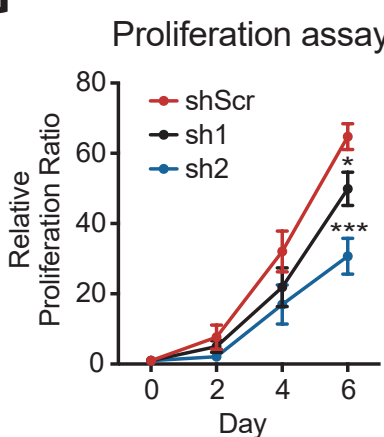

H

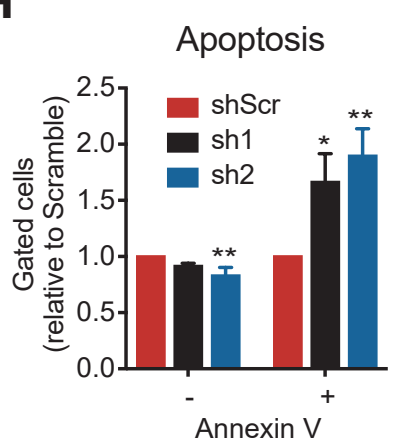

Cell cycle

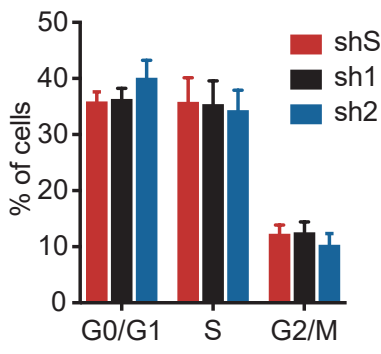


bioRxiv preprint doi: https://doi.or 10.1101/2021.03.02.433507; this versiocposted March 2, 2021. The copyright holder for this prep

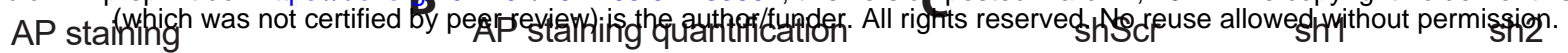

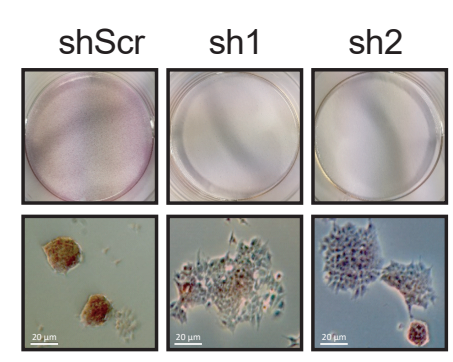

D

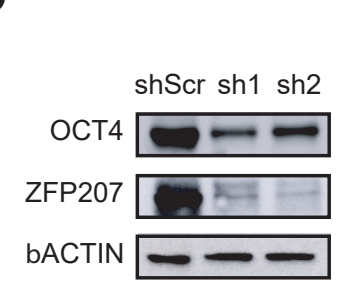

E

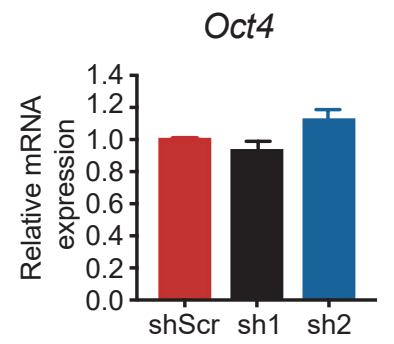

$\mathbf{F}$
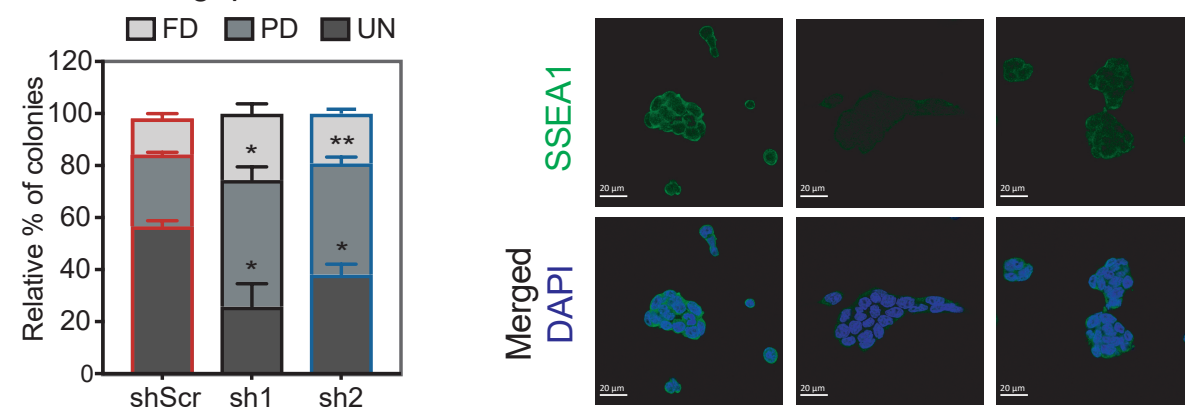

$\mathbf{G}$

$\mathrm{CHX}$ treatment

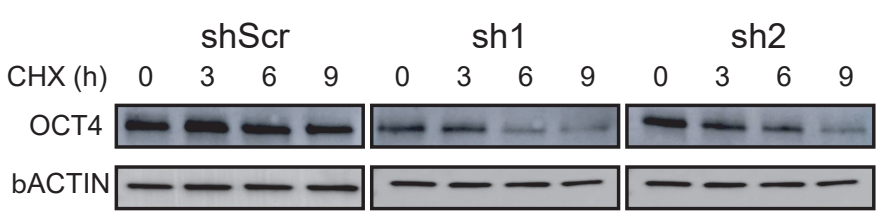

H

MG132 treatment

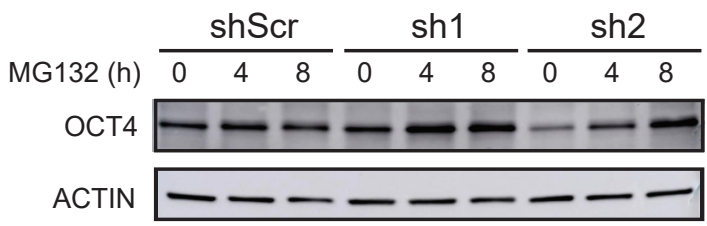

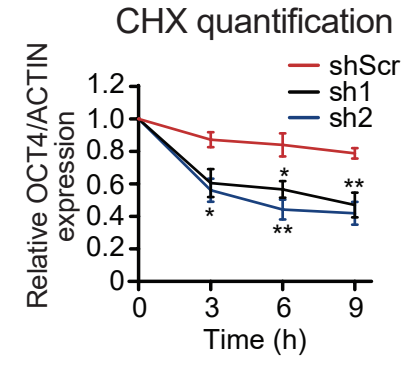

I

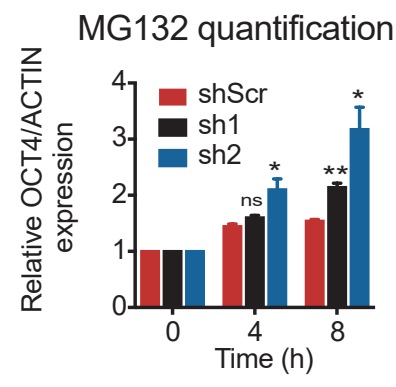


A bioRxiv preprint doi: https://doi.org/10.1101/2021.03.02.433507; this version posted March 2, 2021. The copyright holder for this prep

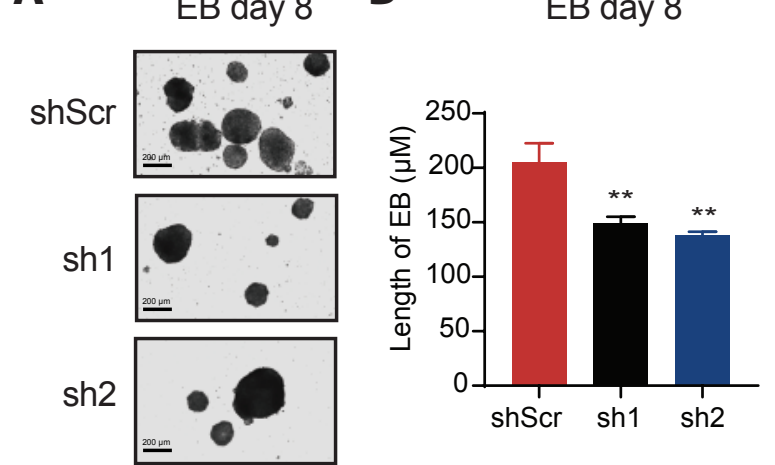

D

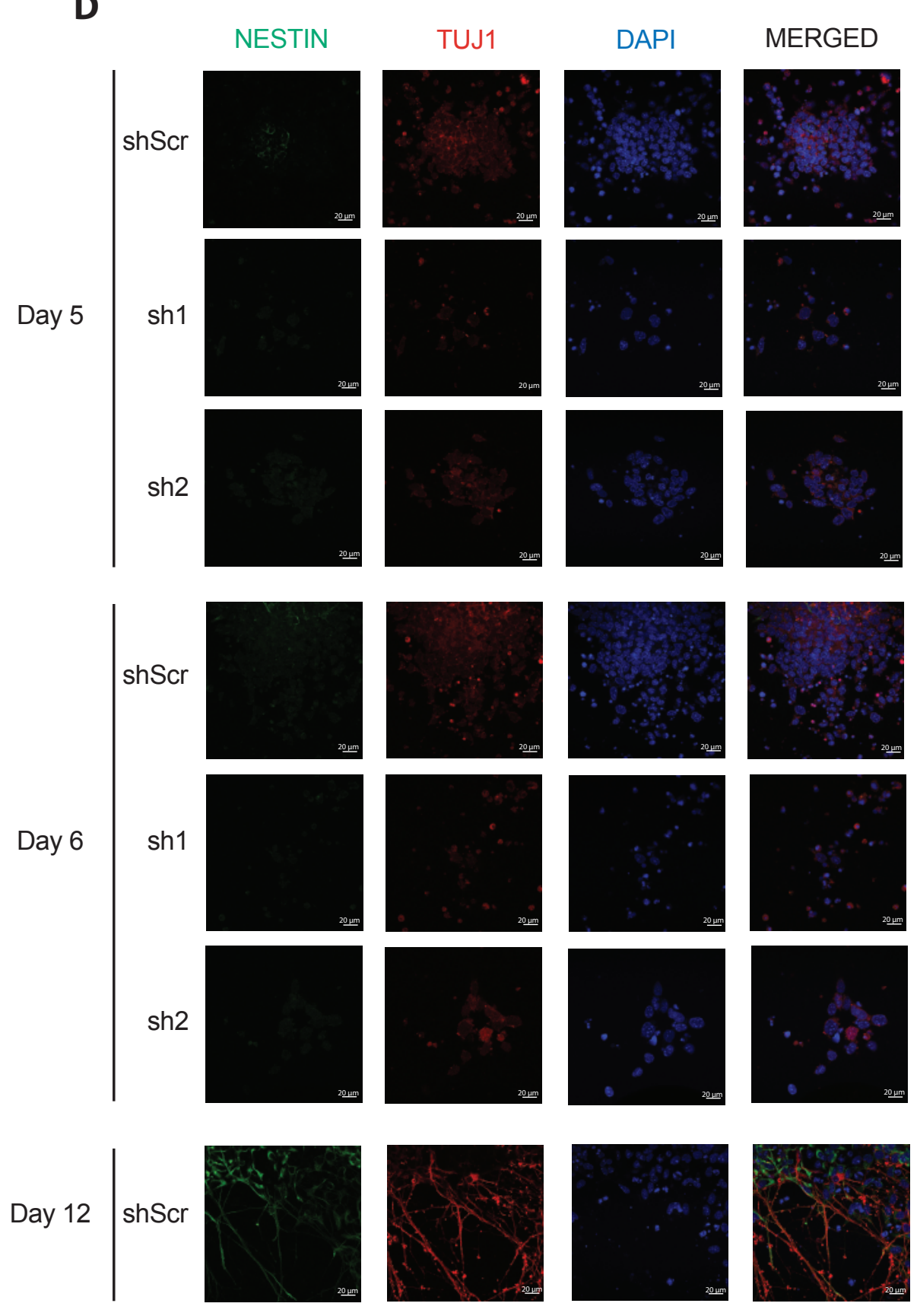

E

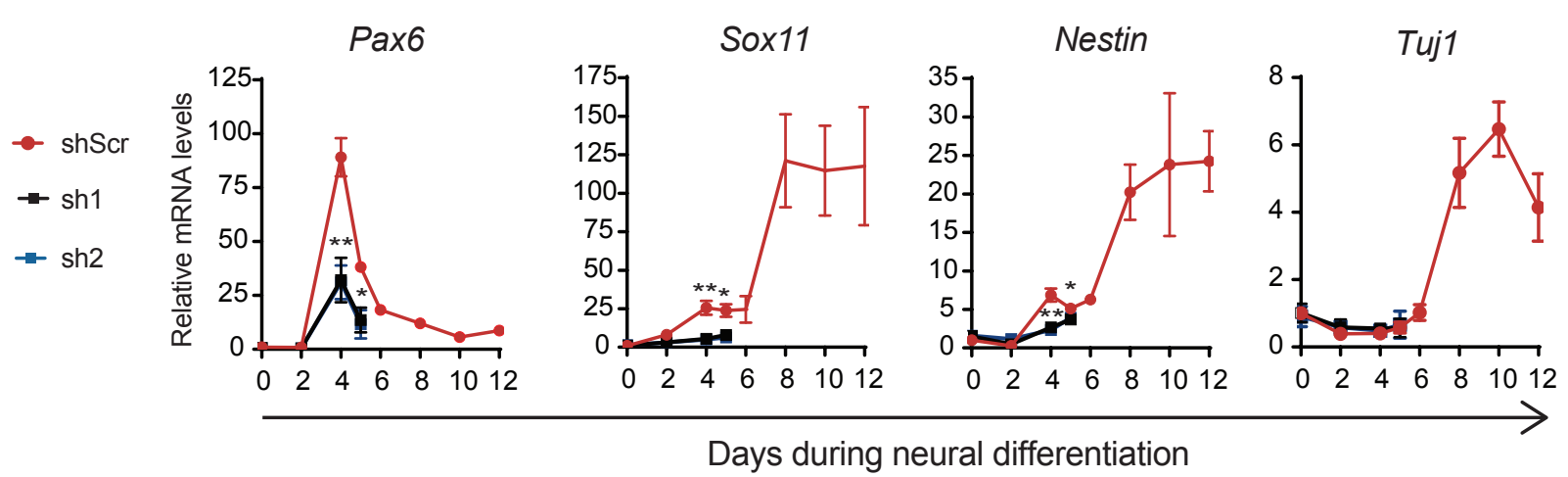


bioRxiv preprint doi: https://doi.org/10.1101/8021.03.02.433507; this version posted March 2, 2021. The copyright holder for this prep (which was not certified by peer review) is the author/funder. All rights reserved. No reuse allowed without permission.

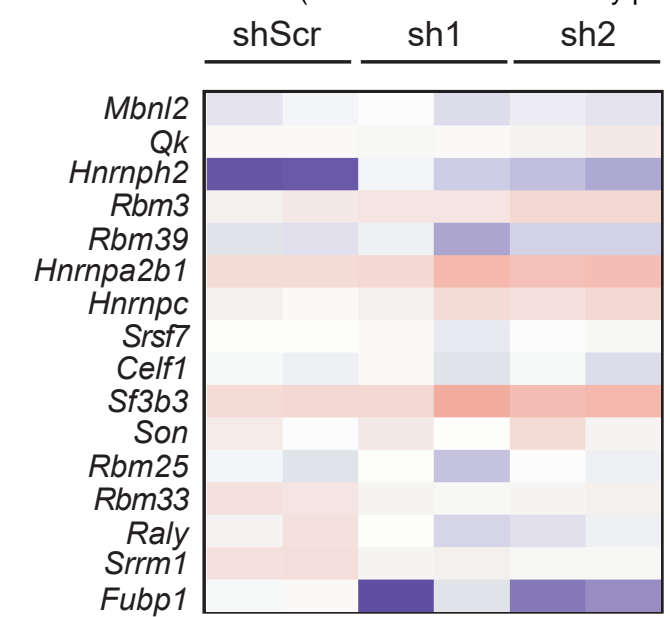

Column Z-score

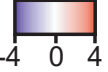

$\mathbf{E}$

GO Biological pathway

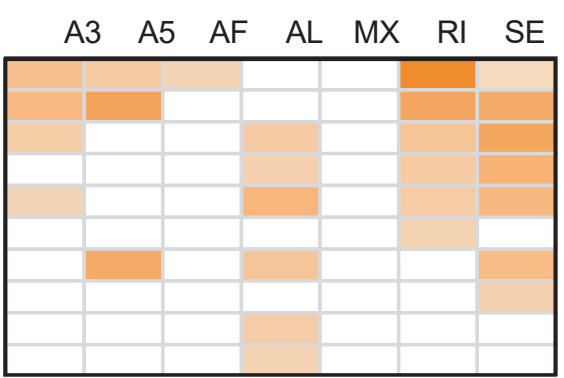

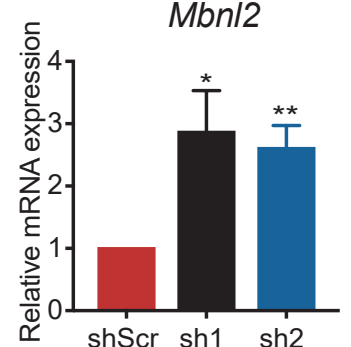

C

shScr vs. shZfp207 (1+2)

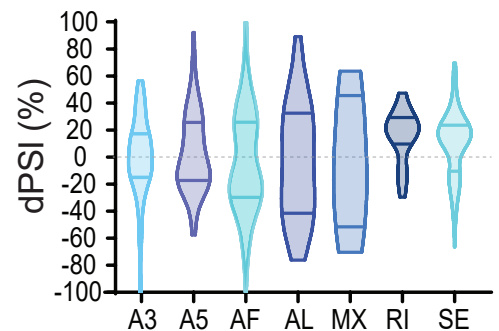

$\mathrm{Rbm3}$

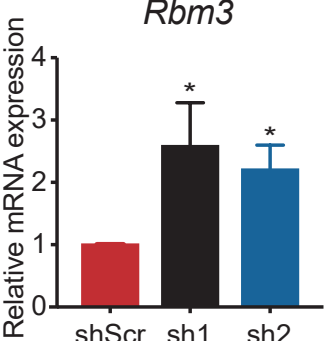

D

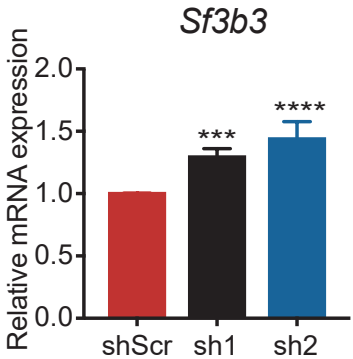

AS events

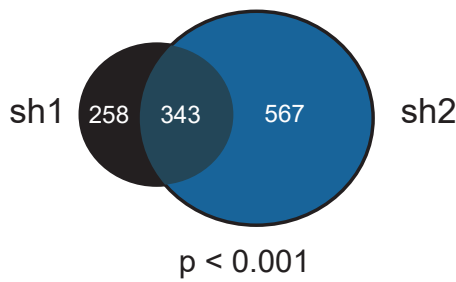

p-value $(-\log 10)$

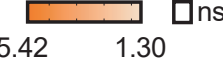

Spliceosomal complex assembly

mRNA splicing, via spliceosome

RNA transport

RNA export from nucleus

RNA export from nucleus

Regulation of synapse organization

Gene expression

Neuron differentiation

Regulation of proteasomal ubiquitin-dependent protein catabolic process

Mitotic spindle midzone assembly

$\mathbf{F}$

\#1

shScr sh1 sh2 shScr sh1 sh2

Mta1 AS1

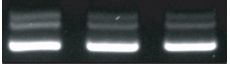

dPSI (\%)

shscr sh1 sh 2 sh 1 sh2

Tbx3

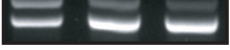

dPSI (\%) $\quad 36 \quad 14 \quad 8$

shScr sh1 sh2 shScr sh1 sh2

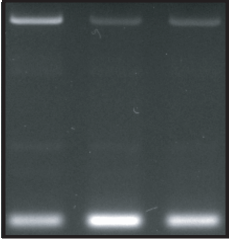

dPSI (\%) $32 \quad 5 \quad 9$

shScr sh1 sh2

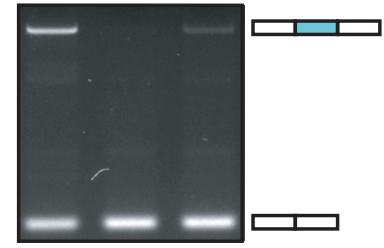

3205

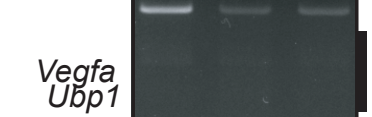

dPSI (\%) $92 \quad 53 \quad 47$

shScr sh1 sh2

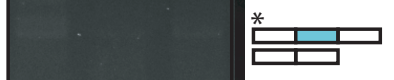

$91 \quad 69 \quad 73$

Fxr1

shScr sh1 sh2 shScr sh1 sh2

dPSI (\%)
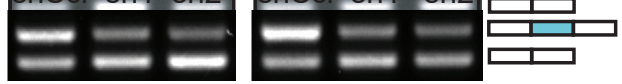

$\begin{array}{lll}63 & 37 & 38\end{array}$

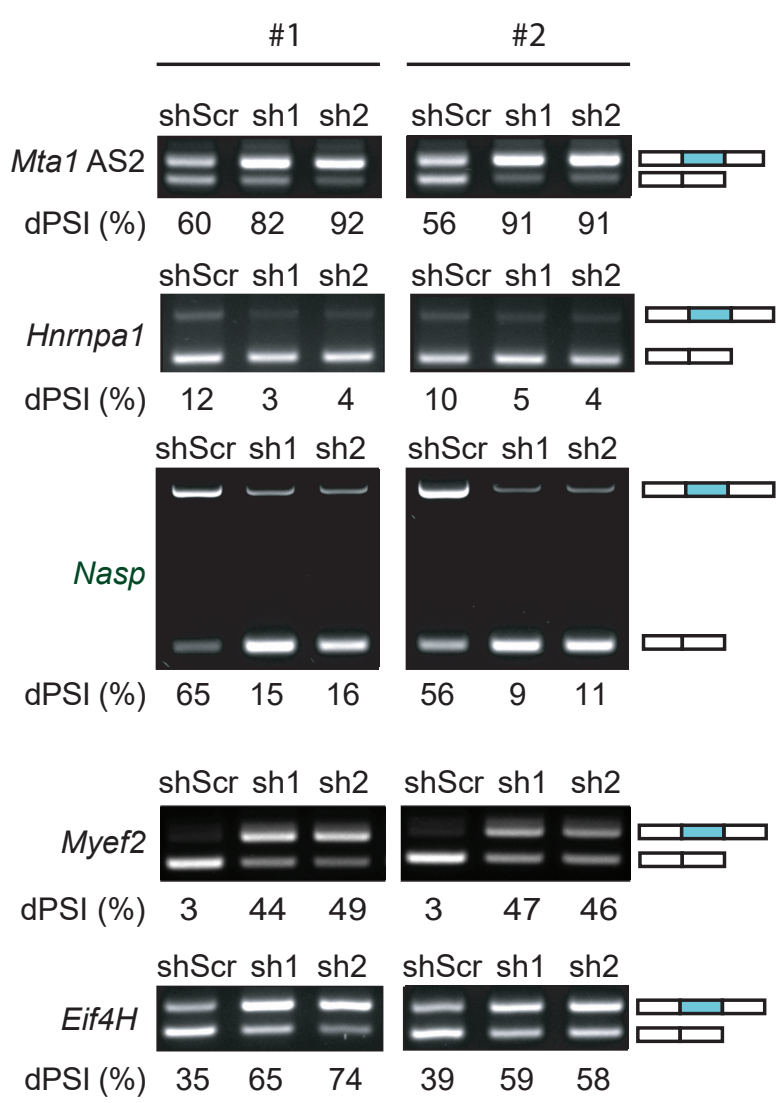


A bioRxiv preprint doi: https://d P.org/10.1101/2021.03.02.433507; this verfon posted March 2, 2021. The copyright holder for this prep (which was not certiffed by peer review) is the author/funder. All Tights reserved. No reuse allowed without permission.

Peak distribution ZFP207-bound transcripts

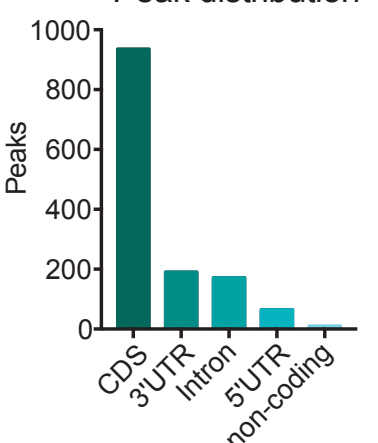

D

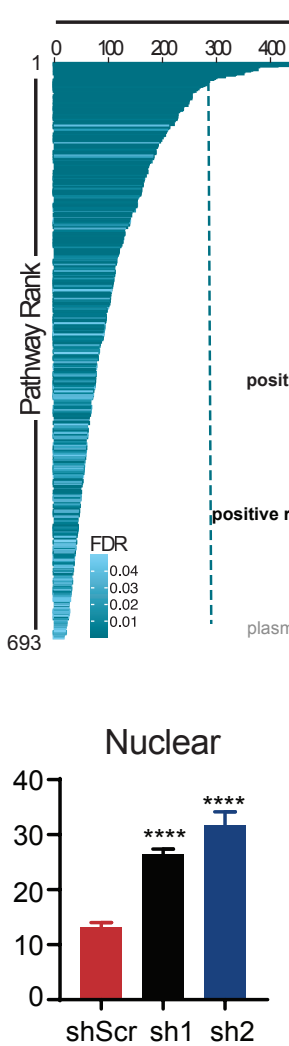

H

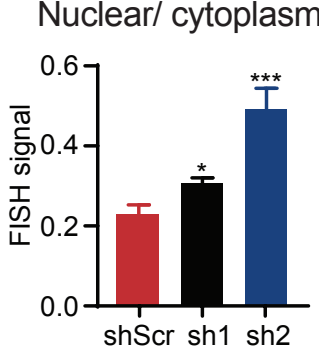

\section{$\mathbf{G}$}

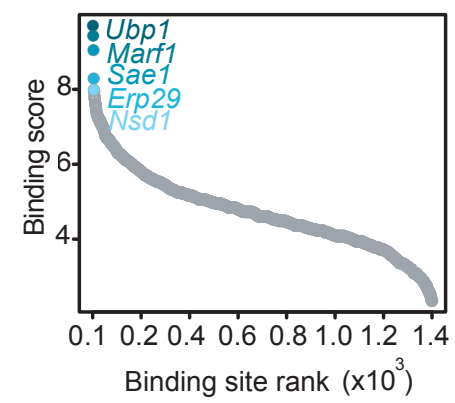

$\mathbf{E}$
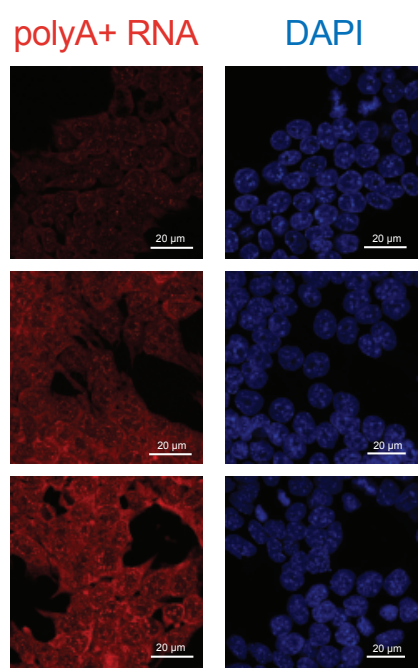

shScr

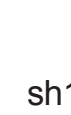

AS

$\operatorname{shZfp207}(1+2)$

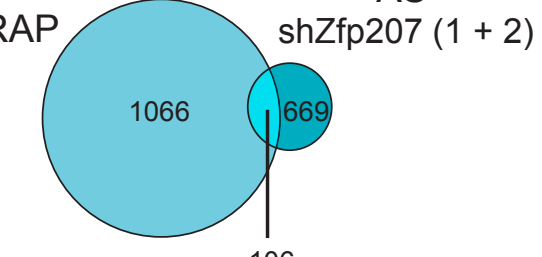

(Mta1, Ubp1, Vegfa, Fxr1)
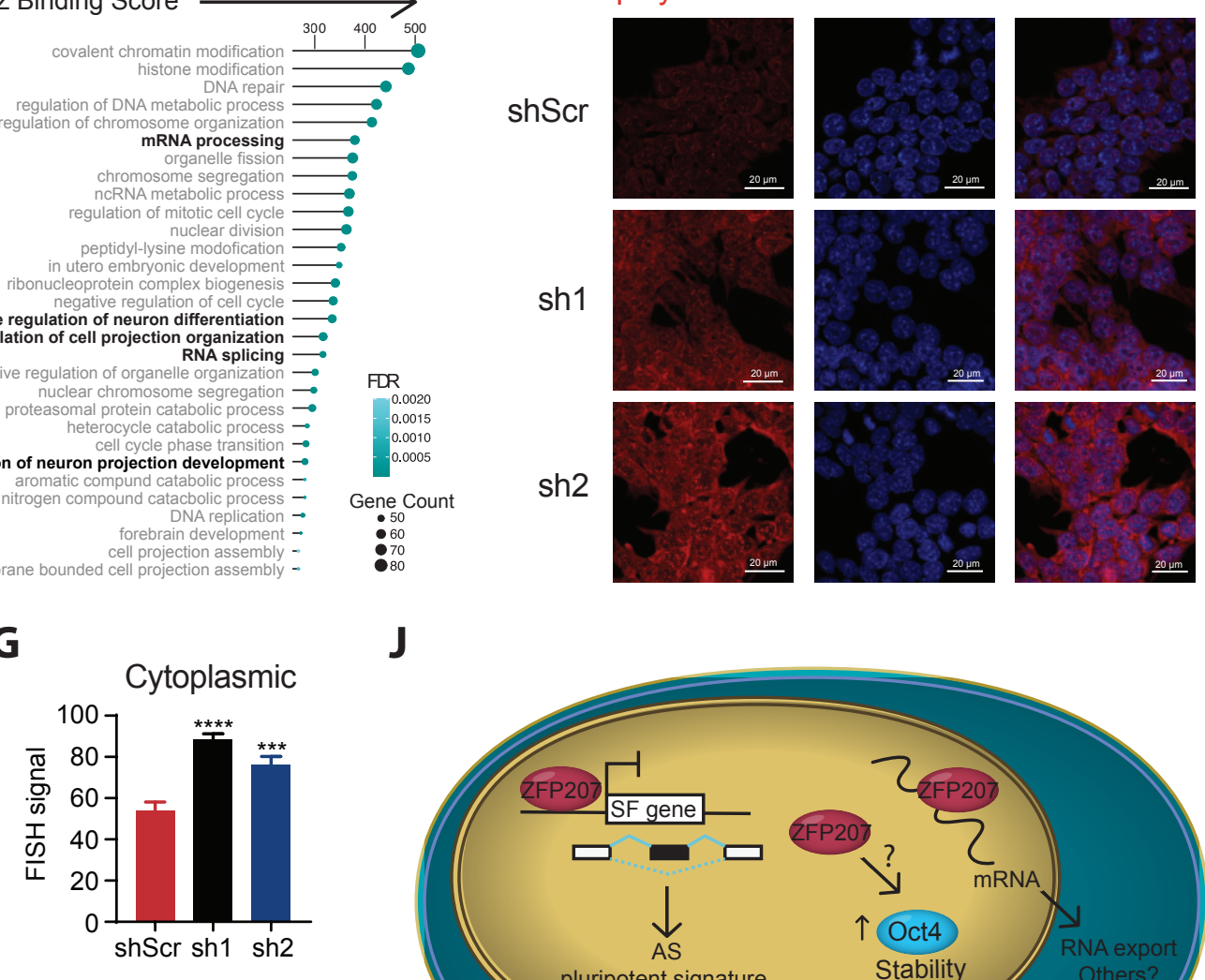

I

Total RNA levels

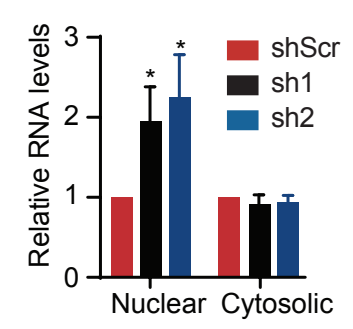

J

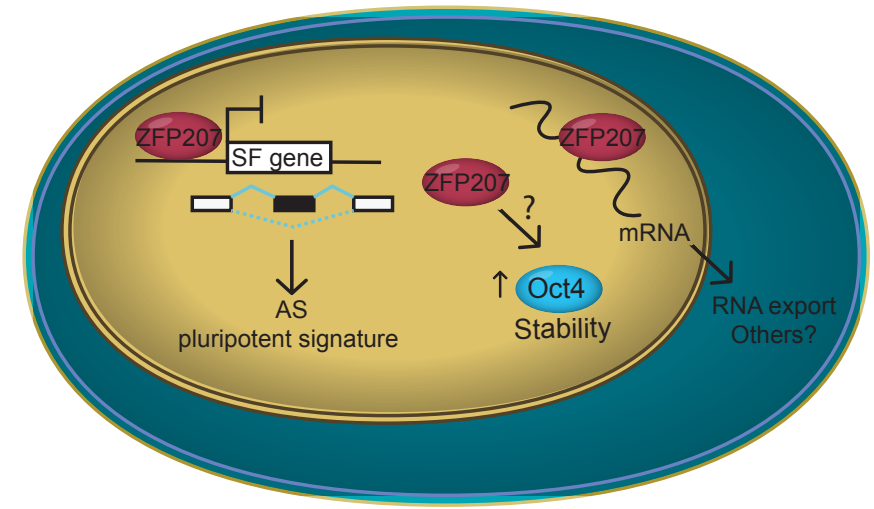

Pluripotency

Neuroectoderm
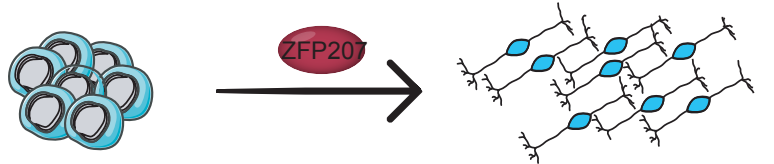